\title{
A GEOMETRIC APPROACH TO MULTIPERIOD MEAN VARIANCE OPTIMIZATION OF ASSETS AND LIABILITIES
}

\author{
MARKUS LEIPPOLD \\ Swiss Banking Institute, University of Zürich \\ FABIO TROJANI \\ Institute of Finance, University of Southern Switzerland \\ PAOLO VANINI \\ Institute of Finance, University of Southern Switzerland
}

\begin{abstract}
We present a geometric approach to discrete time multiperiod mean variance portfolio optimization that largely simplifies the mathematical analysis and the economic interpretation of such model settings. We show that multiperiod mean variance optimal policies can be decomposed in an orthogonal set of basis strategies, each having a clear economic interpretation. This implies that the corresponding multi period mean variance frontiers are spanned by an orthogonal basis of dynamic returns. Specifically, in a $k$-period model the optimal strategy is a linear combination of a single $k$-period global minimum second moment strategy and a sequence of $k$ local excess return strategies which expose the dynamic portfolio optimally to each single-period asset excess return. This decomposition is a multi period version of Hansen and Richard (1987) orthogonal representation of single-period mean variance frontiers and naturally extends the basic economic intuition of the static Markowitz model to the multiperiod context. Using the geometric approach to dynamic mean variance optimization we obtain closed form solutions in the i.i.d. setting for portfolios consisting of both assets and liabilities (AL), each modelled by a distinct state variable. As a special case, the solution of the mean variance problem for the asset only case in $\mathrm{Li}$ and $\mathrm{Ng}$ (2000) follows directly and can be represented in terms of simple products of some single period orthogonal returns. We illustrate the usefulness of our geometric representation of multi-periods optimal policies and mean variance frontiers by discussing specific issued related to AL portfolios: The impact of taking liabilities into account on the implied mean variance frontiers, the quantification of the impact of the investment horizon and the determination of the optimal initial funding ratio.
\end{abstract}

Key Words: Assets and Liabilities Portfolios, Minimum-Variance Frontiers, Dynamic Programming, Markowitz Model

JEL Classification Codes: G11, G12, G28, D92, C60, C61

First version: June 2000. This version: April 2002.

We thank the participants of the 2001 EFMA conference, and seminar participants at the University of Geneva and at Paribas, London, for helpful comments. We also express our gratitude to Giovanni Barone-Adesi, Stefano Galluccio and Robert Tompkins, for stimulating discussions and helpful comments. Fabio Trojani and Paolo Vanini thank the Swiss National Science Foundation (grant nr. 1213-065196.01 and NCCR FINRISK, respectively) for financial support.

Correspondence Information: Fabio Trojani, Institute of Finance, University of Southern Switzerland, Via Buffi 13, 6900 Lugano, Switzerland; e-mail: Fabio.Trojani@lu.unisi.ch. 


\section{INTRODUCTION}

This paper presents a geometric approach to discrete time multiperiod mean variance portfolio optimization that largely simplifies the mathematical analysis and the economic interpretation of such model settings. Using a geometric approach to dynamic mean variance optimization we obtain closed form solutions for an optimization problem where the mean variance objective is defined as a function of the surplus of final aggregate assets and liabilities (AL), each modelled by a distinct state variable. In this setting, the asset-only mean variance problem recently solved in $\mathrm{Li}$ and $\mathrm{Ng}$ (2000) follows as a special solution that can be represented in terms of simple products of single period orthogonal returns. Specifically, we show that multiperiod mean variance optimal policies can be decomposed in an orthogonal set of basis strategies, each having a clear economic interpretation. This implies that the corresponding multi period mean variance frontiers are spanned by an orthogonal basis of dynamic returns. Precisely, in a multi period model the optimal strategy is a linear combination of a single multi period global minimum second moment strategy and a sequence of local excess return strategies which expose the dynamic portfolio optimally to each single-period asset excess return. This decomposition is a multi period version of Hansen and Richard (1987) orthogonal representation of singleperiod mean variance frontiers. This largely simplifies the economic interpretation of the implied dynamic optimal policies and allows to extend the basic intuition of the standard Markowitz model to the multi period setting in a natural way.

A first challenge in multiperiod mean variance portfolio selection derives directly from the definition of the mean variance objective within a dynamic setting, which makes a direct application of dynamic programming techniques cumbersome because of the lack of separability (in the dynamic programming sense) of the mean variance objective. Indeed, some authors

(eg. Chen, Jen, and Zionts (1971)) reported enormous difficulties in solving a pure multiperiod 
mean-variance optimization ${ }^{1}$. Actually, the dynamic mean variance asset only problem has been only recently solved in $\mathrm{Li}$ and $\mathrm{Ng}$ (2000) by embedding mean variance portfolio selection into a mean-second moment portfolio optimization.

A second challenge in multi-period mean variance portfolio choice is related to the financial interpretation behind the optimal policies obtained in a dynamic model. Indeed, while for the asset only case closed form solutions have been provided by $\mathrm{Li}$ and $\mathrm{Ng}$ (2000) under the i.i.d. assumption, the economic structure and the financial interpretation of the implied policies and minimum variance frontiers (MVF) is not transparent and is not directly reconciled with the basic intuition provided by the standard (static) Markowitz solution. The geometric decomposition developed in this paper for the implied optimal policies and MVF identifies the similarities and the differences between the solutions of a static and a dynamic mean variance portfolio selection problem. As in $\mathrm{Li}$ and $\mathrm{Ng}$ (2000) this is achieved by writing the mean variance portfolio selection model as an equivalent mean second moment problem. However, by contrast with their paper we make strong use of orthogonal projections to disentangle the different basis objects and returns arising in the multi period mean variance portfolio optimization. This provides a financial interpretation of the desired optimal policies and mean variance frontiers which is the direct analogue to the static Markowitz model when mean variance frontiers are represented by linear combinations of orthogonal returns as in Hansen and Richard (1987).

A third challenge in multi period mean variance portfolio optimization arises when either intertemporal constraints or further state variables are included in the functional form for the underlying wealth dynamics. In this paper we focus on a particular case of this model class by considering portfolios where liabilities are explicitly included as a second relevant state

\footnotetext{
${ }^{1}$ On the other hand, a large literature has analyzed multiperiod portfolio choice by maximizing some timeadditive expected utility of final wealth and/or multiperiod consumption; see for instance the classical papers of Smith (1967), Chen et al. (1971), Mossin (1968), Merton (1969), Merton (1971) and Samuelson (1969). Work extending the classical Merton $(1969,1971)$ portfolio model is Cvitanic and Karatzas (1992), Grossman and Vila (1992), He and Pages (1993), He and Pearson (1991a), He and Pearson (1991b), Karatzas, Lehoczky, Shreve, and Xu (1991), Shreve and Xu (1992a), Shreve and Xu (1992b), Tepla (1998), Vila and Zariphopoulou (1994), Zariphopoulou (1989).
} 
variable, in excess of the aggregate value of total assets. Assets and liabilities jointly determine the relevant surplus of a financial intermediary. Therefore, this problem is of major relevance for many financial institutions like pension funds or banks where both sides of the balance sheet have to be considered in order to develop an integrated asset liability management. Moreover, as is typically the case in dynamic portfolio optimization, the inclusion of a further state variable drastically enhances the computational complexity in obtaining closed form solutions. Multi period mean variance problems for portfolio of assets and liabilities are not an exception to this rule. However, when adopting our geometric approach to portfolio optimization we are able to provide closed form expressions for the implied solutions and MVF. Furthermore, we can still decompose the associated optimal returns in a way that highlights the economic intuition for each term in the solution. In this decomposition, the asset only solution can be identified as a specific term of the more general assets-and-liabilities optimal portfolio. We illustrate the usefulness of our geometric representation of multi-periods optimal policies and mean variance frontiers by discussing the following specific issues related to AL portfolios: The impact of taking liabilities into account on the implied mean variance frontiers, the impact of the investment horizon and the determination of the optimal initial funding ratio.

The remainder of the paper is organized as follows. Section 2 introduces a set of equivalent AL portfolio selection models. Section 3 derives explicit solutions for the implied optimal policies and MVF frontiers, as well as some orthogonal representations of these objects in terms of some underlying basis returns. Section 4 illustrates the usefulness of our geometric representations by analyzing some specific issues related to AL portfolios while Section 5 concludes.

\section{The Model}

Consider an investor ${ }^{2}$ at time $t=0$ equipped with an initial wealth $x_{0}$ and initial liabilities $l_{0}$. The investor is allowed to rebalance her portfolio over $T$ consecutive transaction periods

\footnotetext{
${ }^{2}$ In the sequel we simply call the financial institution under scrutiny, as for instance a pension fund or the
} treasurer of a bank, the "investor". 
at dates $0,1, \ldots, T-1$. Her objective is to maximize the expected value of the final surplus, i.e. the difference between assets and liabilities at the final date $T$, defined as $S_{T}:=x_{T}-l_{T}$, subject to a variance bound and to some random dynamics for assets and liabilities, specified below.

Without loss of generality but for simplicity of notation we define our model initially using two assets and two liabilities. In a second step we will focus on the exogenous liabilities case which allows for analytical formulas in the i.i.d. setting. Finally, we discuss in a short section the case where the investor can choose from an arbitrary number of assets. It turns out, that when adopting our geometric formalism based on projections the extension from the two assets case to the more general model setting is straightforward.

Given two assets and two liabilities, the first asset return ${ }^{3}\left(R_{t}^{0}\right)$ and the first liability return $\left(Q_{t}^{0}\right)$ are used as benchmark returns for the second asset return $\left(\widetilde{R}_{t}\right)$ and second liability return $\left(\widetilde{Q}_{t}\right)$, respectively. The AL return processes can be cross-sectionally and temporally correlated. We denote the expectation operator by $E$ and the expectation operator conditional on time $t$ by $E_{t}$. Collecting all return processes in the vector $\mathbf{R}_{t}=\left(R_{t}^{0}, \widetilde{R}_{t}, Q_{t}^{0}, \widetilde{Q}_{t}\right)^{\prime}$, we assume the matrices

$$
E_{t}\left(\mathbf{R}_{t} \mathbf{R}_{t}^{\prime}\right)=\operatorname{cov}_{t}\left(\mathbf{R}_{t}\right)+E_{t}\left(\mathbf{R}_{t}\right) E_{t}\left(\mathbf{R}_{t}^{\prime}\right), t=0, . ., T-1
$$

of conditional second moments at time $t$ to be positive definite.

For a given value of aggregate assets $x_{t}$ at time $t, u_{t}$ is defined as the amount invested in the asset return $\tilde{R}_{t}$. The remainder $x_{t}-u_{t}$ is invested in the benchmark return $R_{t}^{0}$. Analogously, given the liabilities $l_{t}$ at time $t$, the amount invested in the liability return $\tilde{Q}_{t}$ is $v_{t}$, while $l_{t}-v_{t}$ is the amount invested in the liability return $Q_{t}^{0}$. Then, the assets and liabilities dynamics can be written as

$$
x_{t+1}=R_{t}^{0} x_{t}+R_{t}^{1} u_{t}, l_{t+1}=Q_{t}^{0} l_{t}+Q_{t}^{1} v_{t}
$$

\footnotetext{
${ }^{3}$ We define the return as the $t+k$-measurable random number $R_{t, t+k}$ for which $R_{t, t+k}=P_{t+k} / P_{t}$ holds, given the price $P$ of a financial instrument. Since we only consider one-period returns, we drop the second time index.
} 
with $R_{t}^{1}=\tilde{R}_{t}-R_{t}^{0}$ and $Q_{t}^{1}=\tilde{Q}_{t}-Q_{t}^{0}$ as the excess return over the benchmark asset and the benchmark liability, respectively.

A standard formulation of the optimization problem of our investor is

$$
(\mathrm{P} 1): \quad\left\{\begin{array}{c}
\max _{u, v} E\left(S_{T}\right) \\
\text { s.t. } \operatorname{var}\left(S_{T}\right) \leq \sigma \text { and }(2.1)
\end{array},\right.
$$

for $\sigma>0$. Problem (P1) covers not only theoretically challenging but also practically relevant cases. For example, situations where

- the number of assets and liabilities is (in principle) arbitrary,

- both assets and liabilities are allowed to be stochastic with arbitrary correlations,

- the implied optimal portfolio can determine optimal positions in the presence of both assets and liabilities,

- the optimal solutions provide a dynamically optimal portfolio strategy.

As an alternative, the portfolio selection problem (P1) can be posed in a different form. It is well known that (P1) is equivalent to problems (P2) and (P3) below:

$$
(\mathrm{P} 2): \quad\left\{\begin{array}{c}
\min _{u, v} \operatorname{var}\left(S_{T}\right) \\
\text { s.t. } E\left(S_{T}\right) \geq \epsilon \text { and }(2.1)
\end{array},\right.
$$

for $\epsilon \geq 0$ and

$$
(\mathrm{P} 3): \quad\left\{\begin{array}{c}
\max _{u, v}\left[E\left(S_{T}\right)-w \operatorname{var}\left(S_{T}\right)\right] \\
\text { s.t. }(2.1)
\end{array},\right.
$$

for some strictly positive risk aversion parameter $w$. Indeed, if $\phi^{*}=\left(u^{*}, v^{*}\right)^{\prime}$ solves (P3), then it solves also (P1) for the final surplus variance implied by $\phi^{*}$ and (P2) for the final surplus expected value implied by $\phi^{*}$. Further, at the solution $\phi^{*}$ of (P3) the identity

$$
w=\frac{\partial E\left(S_{T}\right)}{\partial \operatorname{var}\left(S_{T}\right)}
$$

holds true (see $\mathrm{Li}$ and $\mathrm{Ng}(2000)$ ). As a consequence, from a mathematical point of view we are free in the choice of which problem to solve in order to provide a solution to either $(\mathrm{P} 1)$, $(\mathrm{P} 2)$ or $(\mathrm{P} 3)^{4}$.

\footnotetext{
${ }^{4}$ Notice, however, that (P1)-(P3) are economically not fully equivalent. Indeed, from a more practical viewpoint (P3) is the most demanding one, as it requires the explicit quantification of the investor's trade-off between risk and return (quantified by the parameter $w$ ).
} 
A serious difficulty in solving problems (P1)-(P3) directly, is their non-separability in the sense of dynamic programming. Indeed, it is well-known that conditional expectations satisfy the tower property,

$$
E_{s}\left(E_{t}(\cdot)\right)=E_{s}(\cdot), t>s
$$

while conditional variances do not. Therefore, in the sequel we adopt a more indirect approach that embeds (P1)-(P3) into a new model which is separable. Following $\mathrm{Li}$ and $\mathrm{Ng}$ (2000), the approach for finding a solution to multiperiod mean-variance problems of the form (P1)-(P3) is to introduce an alternative optimization problem (P4) such that:

- The solution of (P4) provides a solution of (P3) (and hence also of (P1) and (P2)).

- $(\mathrm{P} 4)$ is a linear-quadratic optimization problem, i.e. a standard problem in dynamic programming.

It turns out that

$$
(\mathrm{P} 4): \quad\left\{\begin{array}{c}
\max _{u, v}\left[E\left(\lambda S_{T}-w S_{T}^{2}\right)\right] \\
\text { s.t. }(2.1)
\end{array}\right.
$$

satisfies both of the above requirements. When comparing (P3) with (P4), we remark first that (P4) is defined using an extra parameter $\lambda$. Second, (P4) is accessible to dynamic programming because second conditional moments satisfy the tower property (2.4). For the set of solutions to (P3) and (P4), respectively, $\mathrm{Li}$ and $\mathrm{Ng}$ (2000) show for the assets-only case that:

- any solution of (P3) is also a solution of (P4),

- if $\phi^{*}$ is a solution to (P4) for given $\left(\lambda^{*}, w\right)$, then it is also a solution to (P3) for:

$$
\lambda^{*}=1+\left.2 w E\left(S_{T}\right)\right|_{\phi^{*}} .
$$

Therefore, solving (P4) for arbitrary $\lambda$ provides a systematic way of determining the corresponding solution of (P3) by imposing condition (2.5). Remark that in our asset-liability optimization problem the same functional form for the (linear) state dynamics and the (quadratic) objective function as in the asset-only case arise. Therefore, Theorem 1 and 2 in Li and $\mathrm{Ng}$ (2000) can be applied with only slight modifications to cover also mean variance AL 
portfolio problems. For completeness, the next proposition summarizes the relevant results required for the subsequent $\mathrm{AL}$ analysis ${ }^{5}$.

Proposition 2.1. Consider the function

$$
\widetilde{U}\left(E\left(S_{T}\right), E\left(S_{T}^{2}\right)\right)=E\left(S_{T}\right)-w \cdot \operatorname{var}\left(S_{T}\right)
$$

and write $\phi^{*}(w, \lambda)$ for a solution of (P4) and $\phi^{*}(w)$ for a solution of (P3), respectively.

1. $\widetilde{U}$ is a convex function of $E\left(S_{T}\right)$ and $E\left(S_{T}^{2}\right)$.

2. Let

$$
d(\phi, w):=\frac{d \widetilde{U}}{d E\left(S_{T}\right)}=1+\left.2 w E\left(S_{T}\right)\right|_{\phi} .
$$

Then $\phi^{*}(w)$ solves (P4) for a pair $(\lambda, w)$ satisfying $\lambda=d\left(\phi^{*}(w), w\right)$.

3. The condition

$$
\lambda=\lambda^{*}:=d\left(\phi^{*}(w, \lambda), w\right)
$$

is necessary for $\phi^{*}(w, \lambda)$ to be a solution of (P3).

Making use of Proposition 1, we focus in the sequel exclusively on Problem (P4) to provide solutions to the multiperiod AL Problem (P1). Specifically, for computing a solution of (P1) for a specific risk aversion parameter $w$, one first has to provide the solution $\phi^{*}\left(w, \lambda^{*}\right)$ of $(\mathrm{P} 4)$. Moreover, note that the multiperiod MVF implied by (P1) and (P4) are the same, because (P1) and (P4) induce the same solution sets.

\section{Two-Assets Optimization with Exogenous Liabilities}

A special case of Problem (P4) arises in the often relevant case where the structure of liabilities is not under the control of the financial institution under scrutiny, but rather implies a dynamic constraint on the final portfolio's surplus. This problem is a multiperiod version of the static problem in Sharpe and Tint (1990) and Keel and Müller (1995). Thus, in the remainder of the paper we focus on unconstrained $\mathrm{AL}$ optimizations of the general form (P4), where no explicit constraints on the surplus $S_{t}$ at any date $t$ are active and where the liabilities

\footnotetext{
${ }^{5}$ All proofs are in the Appendix. The proof of Proposition 1 is given for completeness.
} 
dynamics are exogenous, i.e. the structure of liabilities cannot be optimized. The methodology presented below can be naturally extended to account for intertemporal portfolio constraints on AL and for endogenous liabilities dynamics (see Leippold, Trojani, and Vanini (2002)). However, such a more general problem will not allow for fully explicit formulas of the implied AL optimal policies and MV frontiers, also in the simplest situation of i.i.d. returns. To express the optimal policies for the AL portfolio problems considered in the paper, the following matrix notation is introduced.

Notation 3.1. Let $\gamma=\frac{\lambda}{2 w}$ and for $t=0, . ., T-1$ define:

$$
\begin{aligned}
& \mathbf{I}=\left(\begin{array}{c}
1 \\
-1
\end{array}\right), D_{t}=\left(\begin{array}{cc}
R_{t}^{0} & 0 \\
0 & Q_{t}^{0}
\end{array}\right), G_{t}=\left(\begin{array}{cc}
R_{t}^{1} & 0 \\
0 & Q_{t}^{1}
\end{array}\right), \\
& z_{t}=\left(\begin{array}{c}
x_{t} \\
l_{t}
\end{array}\right), d_{t}=\left(\begin{array}{c}
u_{t} \\
v_{t}
\end{array}\right) .
\end{aligned}
$$

The matrix $D_{t}$ is the benchmark return matrix and $G_{t}$ the excess return matrix relative to the benchmark for the asset and the liability, respectively. Notice, that the exogenous liabilities case follows, with the above notations, by imposing the constraint $l_{t}=0$ for the amount invested in the second liability return $\tilde{Q}_{t}$, i.e. $d_{t}=\left(u_{t}, 0\right)^{\prime}$. On the other hand, Notation 3.1 can be used without crucial modifications to handle also more general model settings (see again Leippold, Trojani and Vanini (2002)) and allows us to fully exploit the quadratic structure of our AL problems in the proofs presented in the appendix.

\subsection{Basic Problem and General Solution}

By imposing the constraints $v_{T-k}=0, k=1, . ., T$, the relevant optimization problem with exogenous liabilities can be written as:

$$
\left\{\begin{array}{c}
\max _{u}\left[E\left(\gamma S_{T}-S_{T}^{2}\right)\right] \\
\text { s.t. }(2.1), v_{T-k}=0, k=1, . ., T
\end{array} .\right.
$$

Let $e_{1}^{\prime}=(1,0)$ and define recursively for $k=1,2, . ., T$, the matrix sequence of excess returns as

$$
D_{T-k}^{e}=\left[\begin{array}{cc}
R_{T-k}^{0 e} & 0 \\
0 & Q_{T-k}^{0 e}
\end{array}\right]
$$


where

$$
\begin{aligned}
& R_{T-k}^{0 e}=R_{T-k+1}^{0 e} R_{T-k}^{0}-\frac{E_{T-k}\left(R_{T-k+1}^{0 e} R_{T-k}^{1} R_{T-k+1}^{0 e} R_{T-k}^{0}\right)}{E_{T-k}\left[\left(R_{T-k+1}^{0 e} R_{T-k}^{1}\right)^{2}\right]} R_{T-k+1}^{0 e} R_{T-k}^{1}, \\
& Q_{T-k}^{0 e}=Q_{T-k+1}^{0 e} Q_{T-k}^{0}-\frac{E_{T-k}\left(R_{T-k+1}^{0 e} R_{T-k}^{1} Q_{T-k+1}^{0 e} Q_{T-k}^{0}\right)}{E_{T-k}\left[\left(R_{T-k+1}^{0 e} R_{T-k}^{1}\right)^{2}\right]} R_{T-k+1}^{0 e} R_{T-k}^{1},
\end{aligned}
$$

where the initial matrix $D_{T}^{e} \in \mathbb{R}^{2 \times 2}$ is the identity matrix. The optimal AL policy is defined by the quadruplet $\left(x-u_{T-k}^{*}, u_{T-k}^{*}, l_{T-k}, 0\right)$, where the first component is the optimal investment in the benchmark asset, the second one the optimal investment in the second asset and the third one the optimal investment in the benchmark liability. The fourth component is zero by construction in the current setting, so that for the exogenous liabilities problem under scrutiny only $u_{T-k}^{*}$ really needs to be determined. The optimal policy to (3.8) is provided by the next proposition.

Proposition 3.2. Given the optimization problem (3.8), the optimal policy for $k=1,2, . ., T$, equals $\left(x_{T-k}-u_{T-k}^{*}, u_{T-k}^{*}, l_{T-k}, 0\right)$, where

$$
\begin{aligned}
u_{T-k}^{*}= & \gamma \frac{e_{1}^{\prime} E_{T-k}\left(D_{T-k+1}^{e} G_{T-k}\right)^{\prime} \boldsymbol{I}}{e^{\prime} E_{T-k}\left(G_{T-k}^{\prime} D_{T-k+1}^{e^{\prime}} \boldsymbol{I} \boldsymbol{I}^{\prime} D_{T-k+1}^{e} G_{T-k}\right) e_{1}} \\
& -\frac{e_{1}^{\prime} E_{T-k}\left(G_{T-k}^{\prime} D_{T-k+1}^{e^{\prime}} \boldsymbol{I} \boldsymbol{I}^{\prime} D_{T-k+1}^{e} D_{T-k}\right) z_{T-k}}{e^{\prime} E_{T-k}\left(G_{T-k}^{\prime} D_{T-k+1}^{e^{\prime}} \boldsymbol{I} \boldsymbol{I}^{\prime} D_{T-k+1}^{e} G_{T-k}\right) e_{1}} .
\end{aligned}
$$

The optimal policy $u_{T-k}^{*}$ in Proposition 3.2 is a linear combination of a state-independent portfolio weighted by a risk aversion related term (the coefficient $\gamma$ ) and a state-dependent portfolio related to the contemporaneous level of assets and liabilities. The optimal coefficients of these two basis portfolios are described by a function of the second moments in the excess returns matrices $D^{e} G$ and $D^{e} D$. In order to clarify the structure of the optimal policy in Proposition 3.2 we therefore first consider the one- and two-period model setting in some more detail. With the insights gained from this preliminary analysis, we then introduce a geometric symbology based on orthogonal projections which allows us to fully understand the structure of the AL optimal policies and MVF also within a general multiperiod model. 


\subsection{Single-Period Optimization}

For $k=1$, Proposition 3.2 provides the solution to the static problem in Sharpe and Tint (1990) and Keel and Müller (1995). The optimal one-period policy directly follows from (3.10),

$$
\begin{aligned}
u_{T-1}^{*} & =\gamma \frac{e_{1}^{\prime} E\left(G_{T-1}\right)^{\prime} \mathbf{I}}{e_{1}^{\prime} E\left(G_{T-1}^{\prime} \mathbf{I I}^{\prime} G_{T-1}\right) e_{1}}-\frac{e_{1}^{\prime} E\left(G_{T-1}^{\prime} \mathbf{I I}^{\prime} D_{T-1}\right) z_{T-1}}{e_{1}^{\prime} E\left(G_{T-1}^{\prime} \mathbf{I I}^{\prime} G_{T-1}\right) e_{1}} \\
& =\gamma \frac{E\left(R_{T-1}^{1}\right)}{E\left(\left(R_{T-1}^{1}\right)^{2}\right)}-\underbrace{\frac{E\left(R_{T-1}^{1} R_{T-1}^{0}\right) x_{T-1}-E\left(R_{T-1}^{1} Q_{T-1}^{0}\right) l_{T-1}}{E\left(\left(R_{T-1}^{1}\right)^{2}\right)}}_{\text {State Independent }} .
\end{aligned}
$$

The optimal portfolio consists of a state-dependent portfolio yielding a minimum second moment (MSM) surplus at time $T$ (see below) and a state-independent portfolio (weighted by the risk aversion term $\gamma$ ). The implied optimal surplus at time $T$ is

$$
\begin{aligned}
S_{T} & =x_{T-1} R_{T-1}^{0}-l_{T-1} Q_{T-1}^{0}+u_{T-1}^{*} R_{T-1}^{1} \\
& =x_{T-1} R_{T-1}^{0 e}-l_{T-1} Q_{T-1}^{0 e}+\gamma R_{T-1}^{1 e}
\end{aligned}
$$

where

$$
\begin{aligned}
R_{T-1}^{0 e} & =R_{T-1}^{0}-\frac{E\left(R_{T-1}^{1} R_{T-1}^{0}\right)}{E\left(\left(R_{T-1}^{1}\right)^{2}\right)} R_{T-1}^{1}=R_{T-1}^{0}-\frac{\left\langle R_{T-1}^{0}, R_{T-1}^{1}\right\rangle}{\left\langle R_{T-1}^{1}, R_{T-1}^{1}\right\rangle} R_{T-1}^{1}, \\
Q_{T-1}^{0 e} & =Q_{T-1}^{0}-\frac{E\left(R_{T-1}^{1} Q_{T-1}^{0}\right)}{E\left(\left(R_{T-1}^{1}\right)^{2}\right)} R_{T-1}^{1}=Q_{T-1}^{0}-\frac{\left\langle Q_{T-1}^{0}, R_{T-1}^{1}\right\rangle}{\left\langle R_{T-1}^{1}, R_{T-1}^{1}\right\rangle} R_{T-1}^{1}, \\
R_{T-1}^{1 e} & =\frac{E\left(R_{T-1}^{1}\right)}{E\left(\left(R_{T-1}^{1}\right)^{2}\right)} R_{T-1}^{1}=\frac{\left\langle\mathbb{1}, R_{T-1}^{1}\right\rangle}{\left\langle R_{T-1}^{1}, R_{T-1}^{1}\right\rangle} R_{T-1}^{1},
\end{aligned}
$$

We denote by $\langle\cdot, \cdot\rangle$ the scalar product in the space $L_{2}$ of square integrable random variables and by $\mathbb{1}$ the risk free payoff of 1 at time $T$. Hence, the returns $R_{T-1}^{0 e}, Q_{T-1}^{0 e}$ in (3.11) are orthogonal projections of benchmark assets and liability returns on their orthogonal complement relatively to the asset excess return $R_{T-1}^{1}$, while $R_{T-1}^{1 e}$ is an orthogonal projection of the pay-off $\mathbb{1}$ on the asset excess return. This suggests that for an extension of the static result (3.11) to the multiperiod setting a geometric notation based on orthogonal projections would highlight the general structure behind multiperiod AL portfolio problems. We introduce the relevant definitions below. 
Definition 3.3. Let $\mathbb{P}: L_{2} \rightarrow L_{2}$ be an orthogonal projection, that is $\mathbb{P}$ is self-adjoint and $\mathbb{P}^{2}=\mathbb{P}$.

1. We denote by $\mathbb{P}_{M_{T-k}}(X)$ the orthogonal projection of a vector $X_{T-k} \in L_{2}$ on a finite dimensional subspace $M_{T-k} \subset L_{2}$. In particular, we will write $\mathbb{P}_{Y_{T-k}}(X)$ for the orthogonal projection of $X_{T-k}$ on the space spanned by $Y_{T-k} \in L_{2}$.

2. For a subspace $M \subset L_{2}$, we write $M^{\perp}$ for the orthogonal complement of $M$, that is the space of all vectors $Y \in L_{2}$ satisfying $\langle Y, X\rangle=0$ for all $X \in M$. Similarly, $Y^{\perp}$ denotes the orthogonal complement of the vector space generated by $Y$. Hence, for any two vectors $X, Y \in L_{2}$ it follows:

$$
X=\mathbb{P}_{Y}(X)+\mathbb{P}_{Y \perp}(X)
$$

Equipped with these definitions, we rewrite the equations in (3.12) in a more compact form (see Figure 1):

$$
R_{T-1}^{0 e}=\mathbb{P}_{R_{T-1}^{1, \perp}}\left(R^{0}\right), Q_{T-1}^{0 e}=\mathbb{P}_{R_{T-1}^{1, \perp}}\left(Q^{0}\right), R_{T-1}^{1 e}=\mathbb{P}_{R_{T-1}^{1}}(\mathbb{I}) .
$$

In particular, (3.13) stresses the fact that the optimal final surplus $S_{T}$ has been decomposed in (3.11) as a linear combination of two $L_{2}$-orthogonal pay-offs, namely $x_{T-1} \mathbb{P}_{R_{T-1}^{1, \perp}}\left(R^{0}\right)-$ $l_{T-1} \mathbb{P}_{R_{T-1}^{1, \perp}}\left(Q^{0}\right)$ and $\mathbb{P}_{R_{T-1}^{1}}(\mathbb{l})$. The pay-off difference

$$
x_{T-1} \mathbb{P}_{R_{T-1}^{1, \perp}}\left(R^{0}\right)-l_{T-1} \mathbb{P}_{R_{T-1}^{1, \perp}}\left(Q^{0}\right)
$$

is the one-period global MSM final surplus, which is obtained as the difference between a one period assets-only and a one-period liabilities-only MSM pay-off (corresponding to $\gamma=0$, $l_{T-1}=0$, and $\gamma=0, x_{T-1}=0$, respectively). On the other hand, $\mathbb{P}_{R_{T-1}^{1}}(\mathbb{l})$ is the asset excess return which is nearest to the fictive risk-free pay-off $\mathbb{1}$ (in $L_{2}$ norm). Notice, that while the global MSM pay-off $x_{T-1} \mathbb{P}_{R_{T-1}^{1, \perp}}\left(R^{0}\right)-l_{T-1} \mathbb{P}_{R_{T-1}^{1, \perp}}\left(Q^{0}\right)$ is the final surplus of a portfolio with a generally non zero initial position in both assets and liabilities $\left(x_{T-k}, l_{T-k}>0\right)$, the pay-off $\mathbb{P}_{R_{T-1}^{1}}(\mathbb{I})$ is an asset excess return and can be generated by a zero initial cost portfolio investing only in the available asset returns $R_{T-1}^{0}$ and $\tilde{R}_{T-1}$. 


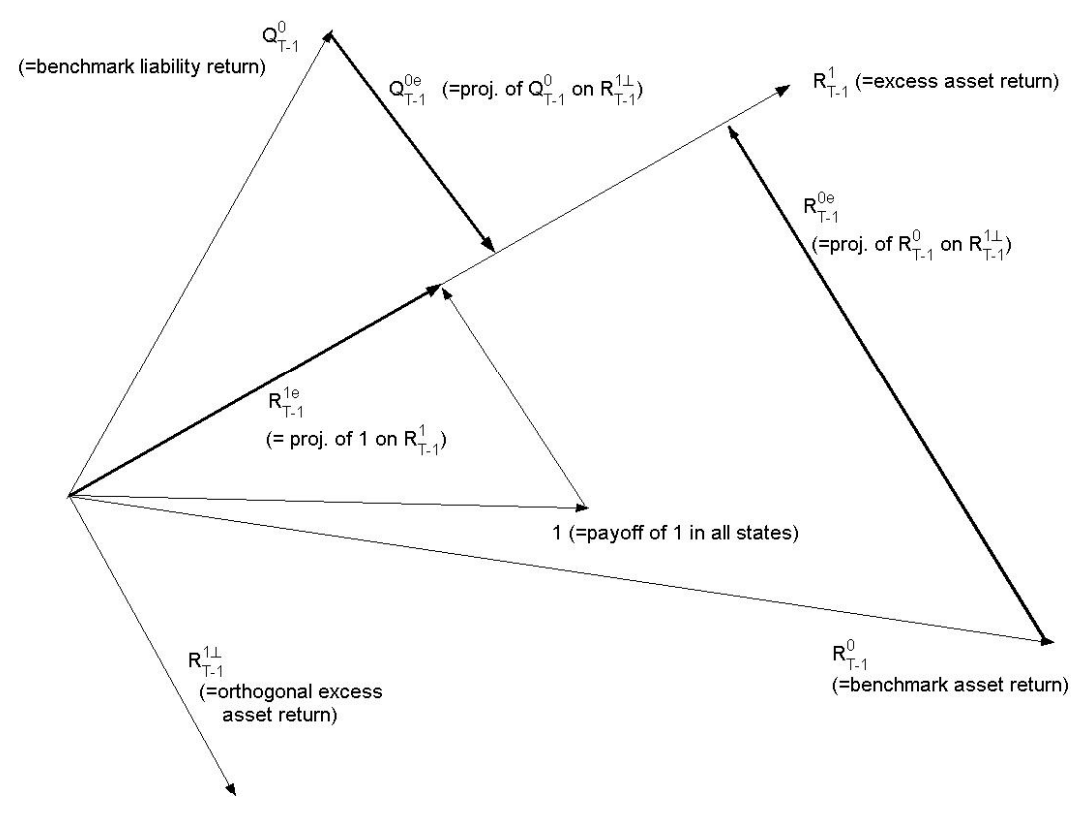

FiguRE 1. Geometry of the projections.

The orthogonal decomposition (3.11) represents the MVF for $S_{T}$, which is defined below for completeness.

Definition 3.4. A final surplus $S_{T}$ belongs to the MVF if its variance is minimal for some targeted final expected surplus.

We set

$$
S_{T-1,0}=x_{T-1} \mathbb{P}_{R_{T-1}^{1, \perp}}\left(R^{0}\right)-l_{T-1} \mathbb{P}_{R_{T-1}^{1, \perp}}\left(Q^{0}\right),
$$

for the MSM surplus and define

$$
\begin{aligned}
\mathcal{A}_{T-1} & =\frac{1}{E\left(\mathbb{P}_{R_{T-1}^{1}}(\mathbb{l})\right)}-1, \\
\mathcal{B}_{T-1} & =\frac{E\left(S_{T-1,0}\right)}{E\left(\mathbb{P}_{R_{T-1}^{1}}(\mathbb{l})\right)}, \\
\mathcal{C}_{T-1} & =\frac{\left[E\left(S_{T-1,0}\right)\right]^{2}}{E\left(\mathbb{P}_{R_{T-1}^{1}}(\mathbb{l})\right)}+E\left(S_{T-1,0}^{2}\right) .
\end{aligned}
$$

The explicit expression for the MVF associated to the static version of problem (3.8) is given in the next proposition. 
Proposition 3.5. Any surplus $S_{T}$ on the $M V F$ is of the form

$$
S_{T}=x_{T-1} \mathbb{P}_{R_{T-1}^{1, \perp}}\left(R^{0}\right)-l_{T-1} \mathbb{P}_{R_{T-1}^{1, \perp}}\left(Q^{0}\right)+\gamma \mathbb{P}_{R_{T-1}^{1}}(\mathbb{I}), \gamma \in \mathbb{R} .
$$

The MVF in $\left(E\left(S_{T}\right), \operatorname{var}\left(S_{T}\right)\right)$-space is given by

$$
\operatorname{var}\left(S_{T}\right)=\mathcal{A}_{T-1} \cdot\left[E\left(S_{T}\right)\right]^{2}-2 \mathcal{B}_{T-1} \cdot E\left(S_{T}\right)+\mathcal{C}_{T-1},
$$

with $\mathcal{A}_{T-1}, \mathcal{B}_{T-1}, \mathcal{C}_{T-1}$, defined in (3.14).

We note that while $\mathcal{B}_{T-1}$ and $\mathcal{C}_{T-1}$ depend on $Q_{T-1}^{0}$, the MVF curvature parameter $\mathcal{A}_{T-1}$ does not. As a consequence, in a static AL optimization with exogenous liabilities the implied MVF if affected by liabilities in only two ways. First, through a "vertical" shift caused by the parameter $\mathcal{C}_{T-1}$ and, second, by a "sidewise" shift caused by the parameter $\mathcal{B}_{T-1}$. Therefore, the introduction of liabilities induces a pure translation of the MVF in the mean-variance space, caused by a modified global MSM surplus $S_{T-1,0}$. The direction of the translation of the AL MVF depends on $Q_{T-1}^{0}$ only through the first and second moments of $S_{T-1,0}$, which can be computed explicitly. A similar structure arises in the multiperiod setting below.

\subsection{Two-Period Optimization}

To clarify the differences between single-period and multiperiod optimizations we consider next the two-period problem. Thanks to the geometric notation (3.13), the structure behind the solution for the more general case will be evident. For $k=2$ the optimal policy is

$$
\begin{aligned}
u_{T-2}^{*}= & \gamma \underbrace{\frac{E_{T-2}\left(R_{T-1}^{0 e} R_{T-2}^{1}\right)}{E_{T-2}\left(\left(R_{T-1}^{0 e} R_{T-2}^{1}\right)^{2}\right)}}_{\text {State Independent }} \\
& -\underbrace{\frac{E_{T-2}\left(R_{T-1}^{0 e} R_{T-2}^{1} R_{T-1}^{0 e} R_{T-2}^{0}\right) x_{T-2}-E_{T-2}\left(R_{T-1}^{0 e} R_{T-2}^{1} Q_{T-1}^{0 e} Q_{T-2}^{0}\right) l_{T-2}}{E_{T-2}\left(\left(R_{T-1}^{0 e} R_{T-2}^{1}\right)^{2}\right)}}_{\text {State Dependent ALM MSM Portfolio }} .
\end{aligned}
$$


Recall that the expressions $R_{T-1}^{0 e}, Q_{T-1}^{0 e}$ contain expectations with respect to information available at time $T-1$. In analogy to the single-period case we decompose the optimal surplus at time $T$ as

$$
\begin{aligned}
S_{T} & =\left(x_{T-2} R_{T-2}^{0}+u_{T-2}^{*} R_{T-2}^{1}\right) R_{T-1}^{0 e}-l_{T-2} Q_{T-2}^{0} Q_{T-1}^{0 e}+\gamma R_{T-1}^{1 e} \\
& =x_{T-2} R_{T-2}^{0 e}-l_{T-2} Q_{T-2}^{0 e}+\gamma\left(R_{T-2}^{1 e}+R_{T-1}^{1 e}\right),
\end{aligned}
$$

where $^{6}$

$$
\begin{aligned}
R_{T-2}^{0 e} & =R_{T-2}^{0} R_{T-1}^{0 e}-\frac{\left\langle R_{T-2}^{1} R_{T-1}^{0 e}, R_{T-2}^{0} R_{T-1}^{0 e}\right\rangle}{\left\langle R_{T-2}^{1} R_{T-1}^{0 e}, R_{T-2}^{1} R_{T-1}^{0 e}\right\rangle} R_{T-2}^{1} R_{T-1}^{0 e}, \\
Q_{T-2}^{0 e} & =Q_{T-2}^{0} Q_{T-1}^{0 e}-\frac{\left\langle R_{T-2}^{1} R_{T-1}^{0 e}, Q_{T-2}^{0} Q_{T-1}^{0 e}\right\rangle}{\left\langle R_{T-2}^{1} R_{T-1}^{0 e}, R_{T-2}^{1} R_{T-1}^{0 e}\right\rangle} R_{T-2}^{1} R_{T-1}^{0 e}, \\
R_{T-2}^{1 e} & =\frac{\left\langle\mathbb{1}, R_{T-2}^{1} R_{T-1}^{0 e}\right\rangle}{\left\langle R_{T-2}^{1} R_{T-1}^{0 e}, R_{T-2}^{1} R_{T-1}^{0 e}\right\rangle} R_{T-2}^{1} R_{T-1}^{0 e} .
\end{aligned}
$$

Comparing (3.16) with (3.12), we see that the returns $R_{T-2}^{0 e}, Q_{T-2}^{0 e}, R_{T-2}^{1 e}$ are more complicated projections than in the static case. Specifically, by Definition 3.3 we have:

$$
\begin{aligned}
& R_{T-2}^{0 e}=\mathbb{P}_{\left(R_{T-2}^{1} R_{T-1}^{0 e}\right)^{\perp}}\left(R_{T-2}^{0} R_{T-1}^{0 e}\right)=\mathbb{P}_{\left(R_{T-2}^{1} R_{T-1}^{0 e}\right)^{\perp}}\left(R_{T-2}^{0} \mathbb{P}_{R_{T-1}^{1, \perp}}\left(R^{0}\right)\right), \\
& Q_{T-2}^{0 e}=\mathbb{P}_{\left(R_{T-2}^{1} R_{T-1}^{0 e}\right)^{\perp}}\left(Q_{T-2}^{0} Q_{T-1}^{0 e}\right)=\mathbb{P}_{\left(R_{T-2}^{1} R_{T-1}^{0 e}\right)^{\perp}}\left(Q_{T-2}^{0} \mathbb{P}_{R_{T-1}^{1, \perp}}\left(Q^{0}\right)\right), \\
& R_{T-2}^{1 e}=\mathbb{P}_{\left(R_{T-2}^{1} R_{T-1}^{0 e}\right)}(\mathbb{l}) .
\end{aligned}
$$

Hence, $\left\{R_{T-2}^{0 e}, Q_{T-2}^{0 e}\right\}$ and $R_{T-2}^{1 e}$ are orthogonal sets of returns. Moreover, by the law of iterated expectations,

$$
\begin{aligned}
& \left\langle R_{T-2}^{1} R_{T-1}^{0 e}, R_{T-1}^{1}\right\rangle=\left\langle R_{T-2}^{1} \mathbb{P}_{R_{T-1}^{1, \perp}}\left(R^{0}\right), R_{T-1}^{1}\right\rangle=0, \\
& \left\langle R_{T-2}^{0} R_{T-1}^{0 e}, R_{T-1}^{1}\right\rangle=\left\langle R_{T-2}^{0} \mathbb{P}_{R_{T-1}^{1, \perp}}\left(R^{0}\right), R_{T-1}^{1}\right\rangle=0, \\
& \left\langle Q_{T-2}^{0} Q_{T-1}^{0 e}, R_{T-1}^{1}\right\rangle=\left\langle Q_{T-2}^{0} \mathbb{P}_{R_{T-1}^{1, \perp}}\left(Q^{0}\right), R_{T-1}^{1}\right\rangle=0,
\end{aligned}
$$

implying

$$
\operatorname{span}\left\{R_{T-2}^{0 e}, Q_{T-2}^{0 e}, R_{T-2}^{1 e}\right\} \perp R_{T-1}^{1} .
$$

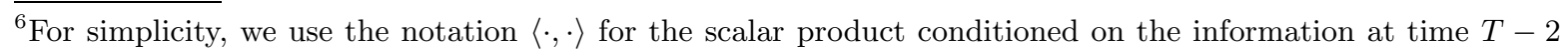
(eg. also Hansen and Richard (1987)). No confusion should occur. 
As a consequence, (3.15) is an orthogonal decomposition of $S_{T}$ in a linear combination of three $L_{2}$-orthogonal returns $x_{T-2} R_{T-2}^{0 e}-l_{T-2} Q_{T-2}^{0 e}, R_{T-2}^{1 e}$ and $R_{T-1}^{1 e}$. Therefore, the same general geometric structure as for the single-period case arises: Single projections are replaced by compositions of projections on suitable subspaces of random variables measurable with respect to information at time $T-1$ and $T-2$, respectively. Precisely, $x_{T-2} R_{T-2}^{0 e}-l_{T-2} Q_{T-2}^{0 e}$ is now the two-period global MSM return for AL portfolios, obtained as the difference between a twoperiod asset-only and a two-period liabilities-only MSM payoff. Further, $R_{T-2}^{1 e}$ is the projection of the final risk free pay-off $\mathbb{l}$ of the space of two-period asset excess returns orthogonal to the final period excess return $R_{T-1}^{1}$. This space is generated by the two-period asset excess return $R_{T-2}^{1} R_{T-1}^{0 e} . \quad R_{T-2}^{1}$ is the asset excess return of a zero initial cost investment from $T-2$ to $T-1$, while $R_{T-1}^{0 e}$ is the return on the one-period MSM asset only portfolio. Therefore, $R_{T-2}^{1 e}$ can be interpreted as the two-period asset excess return of a particular asset-only - zero initial cost - strategy which is exposed to the second asset risk only in the transaction period from $T-2$ to $T-1$. Roughly speaking, this portfolio produces a "local" exposure to the second asset return relatively to the benchmark asset return over the time span from $T-2$ to $T-1$. Therefore, we can interpret $R_{T-2}^{1 e}$ as a "local", two-period, excess asset return. Summarizing, the final surplus $S_{T}$ has been decomposed as the orthogonal sum of a two-period MSM surplus $x_{T-2} R_{T-2}^{0 e}-l_{T-2} Q_{T-2}^{0 e}$ and two "local" asset excess returns $R_{T-2}^{1 e}$ and $R_{T-1}^{1 e}$. An analogous decomposition arises in the general multiperiod setting below.

Since the MVF implied for the two-period model can be represented by a linear combination of orthogonal pay-offs, the same arguments and proofs as for the static case readily apply with comparative statics for the two-period MVF that can be computed explicitly. However, compared to the single period case, a major difficulty shows up. When calculating the MVF, some non-linear nested difference equations for $R^{0 e}, R^{1 e}$ and $Q_{0}^{0 e}$ have to be solved. Considering the difference equations further, it appears that a convolution operator over time characterizes the non-linear part. 
Having clarified the basic geometric structure of the solutions in the simplified two-period setting we now discuss the general multiperiod case.

\subsection{Multiperiod Optimization}

For arbitrary $k$, the optimal policy $u_{T-k}^{*}$ is again a linear combination of a state dependent and a state independent portfolio. We omit the explicit expression corresponding to (3.10), since it follows from the above arguments. Iterating the same arguments as for the two-period model, the orthogonal decomposition generalizing (3.11) and (3.15) to the $k$-period setting is obtained by decomposing the optimal final surplus as

$$
S_{T}=x_{T-k} R_{T-k}^{0 e}-l_{T-k} Q_{T-k}^{0 e}+\gamma \sum_{i=0}^{k-1} R_{T-k+i}^{1 e},
$$

where

$$
\begin{aligned}
R_{T-k}^{0 e} & =\mathbb{P}_{\left(R_{T-k}^{1} R_{T-k+1}^{0 e}\right)^{\perp}}\left(R_{T-k}^{0} R_{T-k+1}^{0 e}\right), \\
Q_{T-k}^{0 e} & =\mathbb{P}_{\left(R_{T-k}^{1} R_{T-k+1}^{0 e}\right)^{\perp}}\left(Q_{T-k}^{0} Q_{T-k+1}^{0 e}\right), \\
R_{T-k+i}^{1 e} & =\mathbb{P}_{\left(R_{T-k+i}^{1} R_{T-k+i+1}^{0 e}\right)}(\mathbb{l}) .
\end{aligned}
$$

Hence, the set of $T$-time pay-offs

$$
\left\{x_{T-k} R_{T-k}^{0 e}-l_{T-k} Q_{T-k}^{0 e}, R_{T-k}^{1 e}, \ldots, R_{T-2}^{1 e}, R_{T-1}^{1 e}\right\}
$$

is an orthogonal system whose span contains the $k$-period MVF. We call the set $\mathbf{B}=\left\{R_{T-k}^{0 e}\right.$, $\left.Q_{T-k}^{0 e}, R_{T-k}^{1 e}, \ldots, R_{T-2}^{1 e}, R_{T-1}^{1 e}\right\}$ a dynamic basis of the model. Recall that for any $j=1, . ., k-$ $1, R_{T-k+j}^{0 e}$ is a $k-j$-period asset only MSM return. Therefore, the return $R_{T-k+i}^{1 e}$ can be interpreted for any $i=0, . ., k-1$ as a "local", $k-i$-period, asset excess return (cf. again the discussion in Section 3.3 for the two-period setting). Summarizing, the final surplus $S_{T}$ is thus decomposed in an orthogonal sum of a $k$-period MSM surplus $x_{T-k} R_{T-k}^{0 e}-l_{T-k} Q_{T-k}^{0 e}$ and $k$ "local" asset excess returns $R_{T-k}^{1 e}, . ., R_{T-1}^{1 e}$. 
Expression (3.17) yields an easily interpretable representation for the multiperiod MVF implied by the underlying model parameters. Indeed, setting

$$
S_{T-k, 0}=x_{T-k} R_{T-k}^{0 e}-l_{T-k} Q_{T-k}^{0 e}, S_{T-k, e}=\sum_{i=0}^{k-1} R_{T-k+i}^{1 e},
$$

and

$$
\mathcal{A}_{T-k}=\frac{1}{E\left(S_{T-k, e}\right)}-1, \mathcal{B}_{T-k}=\frac{E\left(S_{T-k, 0}\right)}{E\left(S_{T-k, e}\right)}, \mathcal{C}_{T-k}=\frac{\left[E\left(S_{T-k, 0}\right)\right]^{2}}{E\left(S_{T-k, e}\right)}+E\left(S_{T-k, 0}^{2}\right)
$$

the implied $k$-period MVF is determined in the next proposition.

Proposition 3.6. Any surplus $S_{T}$ on the k-period $M V F$ is of the form:

$$
S_{T}=S_{T-k, 0}+\gamma S_{T-k, e}, \gamma \in \mathbb{R},
$$

and the MVF in $\left(E\left(S_{T}\right)\right.$, var $\left.\left(S_{T}\right)\right)$-space is given by

$$
\operatorname{var}\left(S_{T}\right)=\mathcal{A}_{T-k} \cdot\left[E\left(S_{T}\right)\right]^{2}-2 \mathcal{B}_{T-k} \cdot E\left(S_{T}\right)+\mathcal{C}_{T-k},
$$

with $\mathcal{A}_{T-k}, \mathcal{B}_{T-k}, \mathcal{C}_{T-k}$ defined in (3.18).

Using the above decomposition of the optimal surplus, the contribution of taking liabilities into account in the assets optimization can be immediately disentangled:

$$
S_{T}=\underbrace{x_{T-k} R_{T-k}^{0 e}+\gamma S_{T-k, e}-l_{T-k} \prod_{i=0}^{k-1} Q_{T-k+i}^{0}}-\underbrace{l_{T-k}\left(Q_{T-k}^{0 e}-\prod_{i=0}^{k-1} Q_{T-k+i}^{0}\right)} .
$$

(A)

(A) is the optimal surplus when neglecting liabilities in the portfolio optimization and (B) is the excess surplus when taking liabilities into account. Whereas (A) depends on the initial levels of assets and liabilities, the moments of the asset return processes as well as the investor's risk aversion, the excess surplus (B) only depends on the initial level of liabilities and the joint moment structure of both assets and liabilities, but not on the risk aversion.

In principle, given a times series model for returns, the pay-offs in (3.19) can be made more explicit in order to obtain the implied optimal policy and the MVF in (3.20). For the i.i.d. benchmark case the following closed-form expressions hold for the dynamic basis $\mathbf{B}$. 
Proposition 3.7. If returns are i.i.d. it follows for $i=0, . ., k-1$ :

$$
\begin{aligned}
R_{T-k}^{0 e} & =\prod_{i=0}^{k-1} \mathbb{P}_{R_{T-k+i}^{1, \perp}}\left(R^{0}\right), \\
R_{T-k+i}^{1 e} & =\mathbb{P}_{R_{T-k+i}^{1}}(\mathbb{I}) \prod_{j=i+1}^{k-1} \mathbb{P}_{\mathbb{P}_{R_{T-k+j}^{1, \perp}}\left(R^{0}\right)}(\mathbb{l}) \\
Q_{T-k}^{0 e} & =\prod_{i=0}^{k-1} Q_{T-k+i}^{0}-\sum_{i=0}^{k-1}\left(\mathbb{P}_{R_{T-k+i}^{1}}\left(Q^{0}\right) \prod_{j=i+1}^{k-1} \mathbb{P}_{\mathbb{P}_{R_{T-k+j}^{1, \perp}}\left(R^{0}\right)}\left(Q^{0}\right) \prod_{j=0}^{i-1} Q_{T-k+j}^{0}\right) .
\end{aligned}
$$

The explicit expressions in Proposition 3.7 can readily be calculated because they are simple products and (or) linear combinations of projections of single-period AL returns. For instance, the return $R_{T-k}^{0 e}$ equals

$$
R_{T-k}^{0 e}=\prod_{i=0}^{k-1} \mathbb{P}_{R_{T-k+i}^{1, \perp}}\left(R^{0}\right)=\prod_{i=0}^{k-1} E\left[R_{T-k+i}^{0} \mid R_{T-k+i}^{1, \perp}\right]
$$

which is a product of prediction errors of $R_{T-k+i}^{0}$ using the instrument $R_{T-k+i}^{1}, i=0, \ldots, k-1$. Given the explicit expressions for the dynamic basis in Proposition 3.7, the explicit structure of the optimal strategy $u_{T-k}^{*}$ is obtained.

COROLLARY 3.8. If returns are i.i.d. the optimal policy $u_{T-k}^{*}$ for problem (3.8) is given by

$$
u_{T-k}^{*}=\gamma u_{T-k}^{1}-\left(u_{T-k}^{x} x_{T-k}-u_{T-k}^{l} l_{T-k}\right),
$$

where

$$
\begin{aligned}
& u_{T-k}^{1}=\frac{\left\langle\mathbb{1}, R_{T-1}^{1}\right\rangle}{\left\langle R_{T-1}^{1}, R_{T-1}^{1}\right\rangle} \cdot \frac{\left\langle\mathbb{1}, \mathbb{P}_{R_{T-1}^{1, \perp}}\left(R^{0}\right)\right\rangle^{k-1}}{\left\langle\mathbb{P}_{R_{T-1}^{1, \perp}}\left(R^{0}\right), \mathbb{P}_{R_{T-1}^{1, \perp}}\left(R^{0}\right)\right\rangle^{k-1}} \\
& u_{T-k}^{x}=\frac{\left\langle R_{T-1}^{0}, R_{T-1}^{1}\right\rangle}{\left\langle R_{T-1}^{1}, R_{T-1}^{1}\right\rangle}, \\
& u_{T-k}^{l}=\frac{\left\langle Q_{T-1}^{0}, R_{T-1}^{1}\right\rangle}{\left\langle R_{T-1}^{1}, R_{T-1}^{1}\right\rangle} \cdot \frac{\left\langle Q_{T-1}^{0}, \mathbb{P}_{R_{T-1}^{1, \perp}}\left(R^{0}\right)\right\rangle^{k-1}}{\left\langle\mathbb{P}_{R_{T-1}^{1, \perp}}\left(R^{0}\right), \mathbb{P}_{R_{T-1}^{1, \perp}}\left(R^{0}\right)\right\rangle^{k-1}}
\end{aligned}
$$

In Corollary 3.8 the part $u_{T-k}^{x} x_{T-k}$ of the optimal policy is proportional to the total assets value $x_{T-k}$ at any time $T-k$. Notice, that the coefficient $u_{T-k}^{x}$ is time-invariant and depends 
only on the (second) moments of the assets return process. Similarly, the part $u_{T-k}^{l} l_{T-k}$ is proportional to the total value of liabilities $l_{T-k}$. However, the coefficient $u_{T-k}^{l}$ is time-dependent and depends on the joint (second) moment structure of the AL return process. Finally, the state independent, but risk-aversion dependent, deterministic assets portfolio $\gamma u_{T-k}^{1}$ is (as $u_{T-k}^{l}$ ) time-variant and depends (as $u_{T-k}^{x}$ ) only on the moments of the assets return process. By contrast to $u_{T-k}^{x}$, in $u_{T-k}^{1}$ both the first and the second moments of the assets return process are present.

Clearly, whenever $u_{T-k}^{l}$ is zero for all $k$, the presence of liabilities does not affect the implied optimal solution. Two cases can be identified for which the optimal portfolio composition is independent of liabilities. The first case requires the scalar product of the benchmark liability return and the excess asset return to be zero, i.e.,

$$
\left\langle Q_{T-1}^{0}, R_{T-1}^{1}\right\rangle=0
$$

The second condition requires the benchmark liability return to be orthogonal to $\mathbb{P}_{R_{T-1}^{1, \perp}}\left(R^{0}\right)$, which is the part of benchmark asset return orthogonal to the excess asset return. Specifically, this boils down the condition

$$
0=\left\langle Q_{T-1}^{0}, R_{T-1}^{0}\right\rangle\left\langle R_{T-1}^{1}, R_{T-1}^{1}\right\rangle-\left\langle R_{T-1}^{0}, R_{T-1}^{1}\right\rangle\left\langle R_{T-1}^{1}, Q_{T-1}^{0}\right\rangle,
$$

which can be interpreted as a singularity condition on the matrix

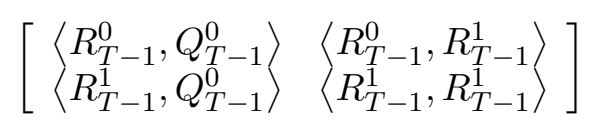

of AL returns second moments. Corollary 3.8 also identifies clear conditions for a myopic strategy to be optimal also in the multiperiod setting. Indeed, from the definition of $u_{T-k}^{1}$ and $u_{T-k}^{l}$ we see that a myopic strategy can be optimal also in the multiperiod model if and only if

$$
\left\langle\mathbb{1}, \mathbb{P}_{R_{T-1}^{1 \perp}}\left(R^{0}\right)\right\rangle=\left\langle Q_{T-1}^{0}, \mathbb{P}_{R_{T-1}^{1 \perp}}\left(R^{0}\right)\right\rangle=\left\langle\mathbb{P}_{R_{T-1}^{1 \perp}}\left(R^{0}\right), \mathbb{P}_{R_{T-1}^{1 \perp}}\left(R^{0}\right)\right\rangle .
$$

As a consequence, for a given arbitrary joint moment structure of assets and liabilities the myopic strategy yields typically a suboptimal dynamic portfolio in the multiperiod context. 
Moreover, the final assets value of an optimal and a myopic strategy can be computed explicitly using our projection formalism, and the differences between the final pay-offs of the two strategies can be clearly identified, as in the next immediate corollary.

COROLlaRY 3.9. If returns are i.i.d., the final asset value for the myopic investor, $x_{T}^{m}$, is given by

$$
\begin{aligned}
x_{T}^{m}= & x_{T-k} \prod_{i=1}^{k} \mathbb{P}_{R_{T-k}^{1 \perp}}\left(R^{0}\right)+l_{T-k} \sum_{i=0}^{k-1}\left(\mathbb{P}_{R_{T-k+i}^{1}}\left(Q^{0}\right) \prod_{j=i+1}^{k-1} \mathbb{P}_{R_{T-k+j}^{1 \perp}}\left(R^{0}\right) \prod_{j=0}^{i-1} Q_{T-k+j}\right) \\
& +\gamma \sum_{i=0}^{k-1}\left(\mathbb{P}_{R_{T-k+i}^{1}}(\mathbb{l}) \prod_{j=i+1}^{k-1} \mathbb{P}_{R_{T-k+j}^{1 \perp}}\left(R^{0}\right)\right),
\end{aligned}
$$

whereas the final asset value implied by a dynamically optimal strategy, $x_{T}^{*}$, is given by

$$
\begin{aligned}
x_{T}^{*}= & x_{T-k} \prod_{i=1}^{k} \mathbb{P}_{R_{T-k}^{1 \perp}}\left(R^{0}\right)+l_{T-k} \sum_{i=0}^{k-1}\left(\mathbb{P}_{R_{T-k+i}^{1}}\left(Q^{0}\right) \prod_{j=i+1}^{k-1} \mathbb{P}_{\mathbb{P}_{R_{T-k+j}^{1 \perp}}\left(R^{0}\right)}\left(Q^{0}\right) \prod_{j=0}^{i-1} Q_{T-k+j}\right) \\
& +\gamma \sum_{i=0}^{k-1}\left(\mathbb{P}_{R_{T-k+i}^{1}}(\mathbb{l}) \prod_{j=i+1}^{k-1} \mathbb{P}_{\mathbb{P}_{R_{T-k+j}^{1 \perp}}\left(R^{0}\right)}(\mathbb{l})\right) .
\end{aligned}
$$

Comparing the final asset values $x_{T}^{m}$ and $x_{T}^{*}$ in Corollary 3.9, the differences arise only because of the different projections $\mathbb{P}_{\mathbb{P}_{R_{T-k+j}^{1 \perp}}}\left(R^{0}\right)\left(R^{0}\right), \mathbb{P}_{\mathbb{P}_{R_{T-k+j}^{1 \perp}}}\left(R^{0}\right)\left(R^{0}\right)$ in $x_{T}^{m}$ and $\mathbb{P}_{\mathbb{P}_{R_{T-k+j}^{1 \perp}}}\left(R^{0}\right)(\mathbb{l})$, $\mathbb{P}_{\mathbb{P}_{R_{T-k+j}^{1 \perp}}}\left(R^{0}\right)\left(Q^{0}\right)$ in $x_{T}^{*}$, respectively. More specifically, the following features hold:

- In the simple case, where there are no liabilities, $l_{T-k}=0$, and the investor is arbitrarily risk averse, i.e. $\gamma \rightarrow 0$, the investor is not concerned about time-diversification effects arising from multiperiod optimization. The myopic strategy coincides with the dynamically optimal strategy. However, as soon as there are non-zero liabilities, the two strategies diverge, even when $\gamma \rightarrow 0$.

- For $x_{T-k} \rightarrow \infty$ ("unbounded wealth"), $l_{T-k} \rightarrow 0$, the accumulation of assets $\left(x_{T}^{m} / x_{T-k}\right.$ and $x_{T}^{*} / x_{T-k}$, respectively) is equal for both the myopic and the dynamically optimal strategy. The final assets return corresponds to the one of the global assets-only MSM strategy. In this case, the investor is indifferent between the two strategies, at least in an approximate way. 
- The difference of the two strategies only enters in the parts, which are dependent on the current value of liabilities $l_{T-k}$ and the risk aversion $\gamma$. However, the part that depends on the current asset value $x_{T-k}$ remains unaffected, i.e. the assets-only MSM portfolio is the same for both strategies.

To study the behavior of the MV frontiers in a general multiperiod AL setting, we also need an explicit expression for the surplus variance in terms of $E\left(S_{T}\right)$. The next corollary accomplishes this task and provides explicit expressions for the implied MVF in the i.i.d. case.

COROLlaRY 3.10. If returns are i.i.d., the k-period MVF in $\left(E\left(S_{T}\right)\right.$, var $\left.\left(S_{T}\right)\right)$-space is given by:

$$
\operatorname{var}\left(S_{T}\right)=\mathcal{A}_{T-k} \cdot\left[E\left(S_{T}\right)\right]^{2}-2 \mathcal{B}_{T-k} \cdot E\left(S_{T}\right)+\mathcal{C}_{T-k}, k=1, . ., T,
$$

where $\mathcal{A}_{T-k}, \mathcal{B}_{T-k}, \mathcal{C}_{T-k}$ are defined in (3.18) and the following explicit expressions hold true:

$$
\begin{aligned}
\left\langle\mathbb{1}, R_{T-k+i}^{1 e}\right\rangle & =\left\langle\mathbb{1}, \mathbb{P}_{R_{T-1}^{1}}(\mathbb{I})\right\rangle\left\langle\mathbb{1}, \mathbb{P}_{\mathbb{P}_{R_{T-1}^{1, \perp}}\left(R^{0}\right)}(\mathbb{I})\right\rangle^{k-i-1}, \\
\left\langle\mathbb{1}, R_{T-k}^{0 e}\right\rangle & =\left\langle\mathbb{1}, \mathbb{P}_{R_{T-1}^{1, \perp}}\left(R^{0}\right)\right\rangle^{k}, \\
\left\langle\mathbb{1}, Q_{T-k}^{0 e}\right\rangle & =\left\langle\mathbb{1}, Q_{T-1}^{0}\right\rangle^{k}-\sum_{i=0}^{k-1}\left\langle\mathbb{1}, Q_{T-1}^{0}\right\rangle^{i}\left\langle\mathbb{1}, \mathbb{P}_{R_{T-1}^{1}}\left(Q^{0}\right)\right\rangle\left\langle\mathbb{1}, \mathbb{P}_{\mathbb{P}_{R_{T-1}^{1, \perp}\left(R^{0}\right)}}\left(Q^{0}\right)\right\rangle^{k-i-1},
\end{aligned}
$$

and

$$
\begin{aligned}
\left\langle R_{T-k}^{0 e}, R_{T-k}^{0 e}\right\rangle= & \left\langle R_{T-1}^{0}, \mathbb{P}_{R_{T-1}^{1, \perp}}\left(R^{0}\right)\right\rangle^{k}, \\
\left\langle Q_{T-k}^{0 e}, R_{T-k}^{0 e}\right\rangle= & \left\langle Q_{T-1}^{0}, \mathbb{P}_{R_{T-1}^{1, \perp}}\left(R^{0}\right)\right\rangle^{k}, \\
\left\langle Q_{T-k}^{0 e}, Q_{T-k}^{0 e}\right\rangle= & \left\langle Q_{T-1}^{0}, Q_{T-1}^{0}\right\rangle^{k} \\
& -\sum_{i=0}^{k-1}\left\langle Q_{T-1}^{0}, Q_{T-1}^{0}\right\rangle^{i}\left\langle Q_{T-1}^{0}, \mathbb{P}_{R_{T-1}^{1}}\left(Q^{0}\right)\right\rangle\left\langle Q_{T-1}^{0}, \mathbb{P}_{\mathbb{P}_{R_{T-1}^{1, \perp}}\left(R^{0}\right)}\left(Q^{0}\right)\right\rangle^{k-i-1} .
\end{aligned}
$$

Since all required scalar products in Corollary 3.10 can be expressed as sums and powers of simple scalar products of single-period returns and projections of single period returns, computation of the desired multiperiod frontiers becomes a straightforward task in applications. In Section 4 we illustrate the usefulness of the representation in Corollary 3.10 by quantifying 
the impact of the investment horizon on the AL multiperiod MVF in some numerical examples and by characterizing analytically the set of inial funding ratios.

\subsection{Several Assets}

When using the geometric projections formalism introduced above, the generalization of the solutions for a two assets-one liability model to a general setting with an arbitrary number of assets becomes a straightforward task. In this section we briefly show how this can be achieved without modifying the basic geometric structure of the previous sections.

First, we extend the asset return processes to an $\mathbb{R}^{(n+1)}$-valued process given by:

$$
\left(R_{t}^{0}, \tilde{R}_{t}^{1}, \tilde{R}_{t}^{2}, . ., \tilde{R}_{t}^{n}\right)_{t=0, . ., T-1}^{\prime}
$$

Then, we define by $\mathcal{R}_{t}=\left(R_{t}^{1}, R_{t}^{2}, . ., R_{t}^{n}\right)$ the row vector of the extended matrix of assets excess returns,

$$
\mathcal{R}=\left(R_{j}^{i}\right)_{1 \leq i \leq n, 0 \leq j \leq T-1},
$$

with $R_{j}^{i}=\tilde{R}_{j}^{i}-R_{j}^{0}$. By $u_{t}^{i}$ we denote the amount of current total assets value $x_{t}$ that is allocated to the asset with return $\tilde{R}_{t}^{i}$ at time $t$. The implied assets and liabilities dynamics for the multi-assets case is

$$
x_{t+1}=R_{t}^{0} x_{t}+\mathcal{R}_{t} u_{t}, l_{t+1}=Q_{t}^{0} l_{t}+Q_{t}^{1} v_{t},
$$

with $u_{t}^{\prime}=\left(u_{t}^{1}, . ., u_{t}^{n}\right)$. Hence, the state dynamics for assets and liabilities can be written in matrix form as

$$
z_{t+1}=D_{t} z_{t}+G_{t} d_{t}
$$

with $D_{t}$ defined in Notation 3.1 and $G_{t}, d_{t}$ given by

$$
G_{t}=\left[\begin{array}{cc}
\mathcal{R}_{t} & 0 \\
0_{1 \times n} & Q_{t}^{1}
\end{array}\right], d_{t}=\left(\begin{array}{c}
u_{t} \\
v_{t}
\end{array}\right) .
$$

Formally, the relevant multiperiod portfolio problem is of the same general form as in (3.8):

$$
\left\{\begin{array}{c}
\max _{u, v}\left[E\left(\gamma S_{T}-S_{T}^{2}\right)\right] \\
\text { s.t. }(2.1), v_{T-k}=0, k=1, . ., T
\end{array},\right.
$$


with the only difference that now the control variable $u$ is a vector valued process. Define now recursively for $k=1,2, . ., T$, the following matrix sequence of excess returns

$$
D_{T-k}^{e}=\left[\begin{array}{cc}
R_{T-k}^{0 e} & 0 \\
0 & Q_{T-k}^{0 e}
\end{array}\right]
$$

where

$$
\begin{aligned}
R_{T-k}^{0 e}= & R_{T-k}^{0} R_{T-k+1}^{e 0} \\
& -\mathcal{R}_{T-k} R_{T-k+1}^{e 0}\left[E\left(\mathcal{R}_{T-k}^{\prime} R_{T-k+1}^{e 0} \mathcal{R}_{T-k} R_{T-k+1}^{e 0}\right)\right]^{-1} E\left(\mathcal{R}_{T-k}^{\prime} R_{T-k+1}^{e 0} R_{T-k}^{0} R_{T-k+1}^{e 0}\right), \\
Q_{T-k}^{0 e}= & Q_{T-k+1}^{e 0} Q_{T-k}^{0} \\
& -\mathcal{R}_{T-k} R_{T-k+1}^{e 0}\left[E\left(\mathcal{R}_{T-k}^{\prime} R_{T-k+1}^{e 0} \mathcal{R}_{T-k} R_{T-k+1}^{e 0}\right)\right]^{-1} E\left(\mathcal{R}_{T-k}^{\prime} R_{T-k+1}^{e 0} Q_{T-k}^{0} Q_{T-k+1}^{e 0}\right),
\end{aligned}
$$

for an initial identity matrix $D_{T}^{e} \in \mathbb{R}^{2 \times 2}$. With these definitions, the optimal policy to the $n$ assets - one liability problem in (3.23) is immediately obtained as for the simpler case analyzed in the previous sections.

Proposition 3.11. For $k=1,2, . ., T$, the optimal policy $d_{T-k}^{* \prime}=\left(u_{T-k}^{*^{\prime}}, 0\right)$ for problem (3.23) is given by:

$$
\begin{aligned}
u_{T-k}^{*}= & \gamma\left[E\left(\mathcal{R}_{T-k}^{\prime} R_{T-k+1}^{0 e} \mathcal{R}_{T-k} R_{T-k+1}^{0 e}\right)\right]^{-1} E\left(\mathcal{R}_{T-k}^{\prime} R_{T-k+1}^{0 e}\right) \\
& -\left[E\left(\mathcal{R}_{T-k}^{\prime} R_{T-k+1}^{0 e} \mathcal{R}_{T-k} R_{T-k+1}^{0 e}\right)\right]^{-1} E\left(\mathcal{R}_{T-k}^{\prime} R_{T-k+1}^{0 e} \boldsymbol{I}^{\prime} D_{T-k} D_{T-k+1}^{e}\right) z_{T-k} .
\end{aligned}
$$

For the implied optimal surplus at time $T$ it follows:

$$
S_{T}=R_{T-k}^{0 e} x_{T-k}+Q_{T-k}^{0 e} l_{T-k}+\gamma \sum_{i=0}^{k-1} \mathbb{P}_{M_{T-k+i}}(\mathbb{l}),
$$

where $M_{T-k+i}$ is the linear span of the vector $\mathcal{R}_{T-k+i}$ weighted by the orthogonal returns $R_{T-k+i+1}^{0 e}$. Following the same arguments as for the case with two assets, the same geometric structure for the optimal portfolio policy arises.

COROLLARY 3.12. If returns are i.i.d. the optimal policy $d_{T-k}^{* \prime}=\left(u_{T-k}^{*^{\prime}}, 0\right)$ for problem (3.23) is given by:

$$
u_{T-k}^{*}=\gamma u_{T-k}^{1}-\left(u_{T-k}^{x} x_{T-k}-u_{T-k}^{l} l_{T-k}\right)
$$


where

$$
\begin{aligned}
u_{T-k}^{1} & =\left[E\left(\mathcal{R}_{T-1}^{\prime} \mathcal{R}_{T-1}\right)\right]^{-1} E\left(\mathcal{R}_{T-1}^{\prime} \mathbb{l}\right) \cdot \frac{\left\langle\mathbb{1}, \mathbb{P}_{M_{T-1}^{\perp}}\left(R^{0}\right)\right\rangle^{k-1}}{\left\langle\mathbb{P}_{M_{T-1}^{\perp}}\left(R^{0}\right), \mathbb{P}_{M_{T-1}^{\perp}}\left(R^{0}\right)\right\rangle^{k-1}}, \\
u_{T-k}^{x} & =\left[E\left(\mathcal{R}_{T-1}^{\prime} \mathcal{R}_{T-1}\right)\right]^{-1} E\left(\mathcal{R}_{T-1}^{\prime} R_{T-1}^{0}\right), \\
u_{T-k}^{l} & =\left[E\left(\mathcal{R}_{T-1}^{\prime} \mathcal{R}_{T-1}\right)\right]^{-1} E\left(\mathcal{R}_{T-1}^{\prime} Q_{T-1}^{0}\right) \cdot \frac{\left\langle Q_{T-1}^{0}, \mathbb{P}_{M_{T-1}^{\perp}}\left(R^{0}\right)\right\rangle^{k-1}}{\left\langle\mathbb{P}_{M_{T-1}^{\perp}}\left(R^{0}\right), \mathbb{P}_{M_{T-1}^{\perp}}\left(R^{0}\right)\right\rangle^{k-1}} .
\end{aligned}
$$

and $\mathbb{P}_{M_{T-1}}$ denotes the projection on the linear span generated by $\mathcal{R}_{T-1}$.

As expected, the same basic geometric structure as for the two assets case arises. Specifically, $u_{T-k}^{x} x_{T-k}$ is now a vector of allocations proportional to the current assets value $x_{T-k}$, with weights $u_{T-k}^{x}$ that are time invariant and that depend only on the joint second moment structure of assets returns. The portfolio part $u_{T-k}^{l} l_{T-k}$ is defined by time varying weights $u_{T-k}^{l}$ that depend on the joint second moment structure of assets and liabilities. Finally, $u_{T-k}^{1}$ depends again on the first two moments of assets returns. Moreover, notice that in the time varying coefficients defining the time varying part of $u_{T-k}^{l}$ and $u_{T-k}^{1}$, projections are now on the space orthogonal to all assets excess returns.

Using Corollary 3.12 we can easily derive for the implied AL-MVF analogous features as the ones proved for the simple two-assets model. We omit the corresponding proofs and results since they are identical to those presented in the previous sections (cf. for instance Proposition 3.6), basically only with projections on the space spanned by $R_{T-1}^{1}$ in the two assets model that are replaced by projections on the space spanned by the $n-1$ dimensional set $\mathcal{R}_{T-1}$ in the multi assets context.

\section{Applications}

In this section we illustrate the usefulness of a geometric approach to multiperiod mean variance portfolio selection by analyzing in some numerical example a few specific issues related to the multi period optimization of AL portfolios. We illustrate (i) the time diversification effects arising through a dynamic policy selection, (ii) the impact of the investment horizon 
on the dynamically optimal portfolio weights, and (iii) the determination of an optimal initial funding ratio for AL portfolios.

\subsection{Time Diversification and MVF}

We consider a model with i.i.d. returns and an investment horizon of one year split into $h$ transaction periods. We compare the MVF implied by a dynamically optimal investment strategy and the one implied by a static strategy that fixes the optimal portfolio once at the beginning of the investment period. This helps in quantifying the time diversification effects deriving from adopting a dynamically optimal AL strategy in mean variance portfolio selection, given an a priori moment structure of the AL return process.

The dynamic strategy rebalances the portfolio $h$ times according to the optimal portfolio weights calculated by the dynamically optimal multiperiod strategy of the previous sections (cf. Corollary 3.8) and uses $h$ i.i.d returns with the associated first and second moments to model the implied AL dynamics. By contrast, the static strategy optimizes the portfolio once at the beginning of the investment horizon and based on yearly returns with the associated first and second moments. In this case no portfolio rebalancing occurs after the initial date.

We fix the inial assets and liabilities as $x_{0}=l_{0}=1$. The vector of expected returns of the two assets and the benchmark liability, respectively, is $\mu=\left(\mu_{1}, \mu_{2}, \mu_{3}\right)^{\prime}$, so that the expected excess asset return, $E\left(R^{1}\right)$, is given by $\mu_{2}-\mu_{1}$. In our examples the vector of yearly mean returns is

$$
\mu=(1.03,1.13,1.04)^{\prime}
$$

The matrix of second moments returns is obtained from a yearly covariance matrix $\Sigma$ given by

$$
\Sigma=\left(\begin{array}{lll}
0.0040 & 0.0026 & 0.0006 \\
0.0026 & 0.0589 & 0.0149 \\
0.0006 & 0.0149 & 0.0100
\end{array}\right)
$$

To obtain the relevant moment matrices for a dynamic strategy optimizing over $h$ transaction periods we first write each yearly return as $R=\prod_{i=0}^{h} R_{i}$ and then compute the relevant moments using the i.i.d assumption. Figure 2 plots the implied MVF for $h=1,2,12,360$. 


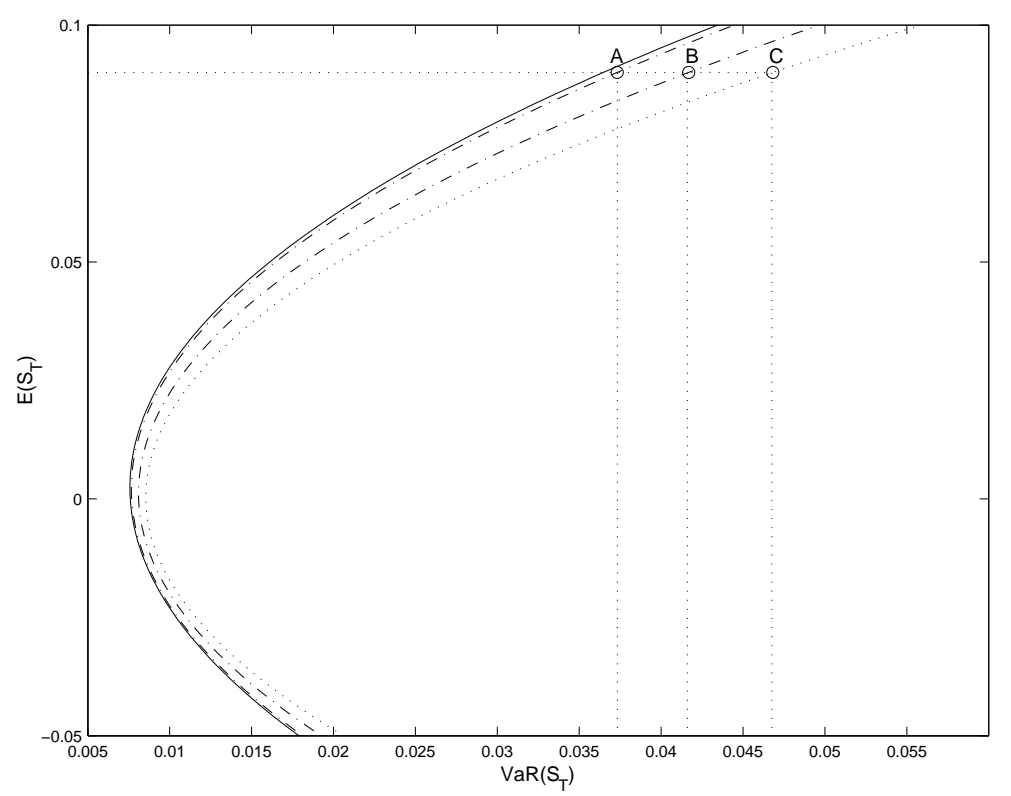

Figure 2. Dynamically Optimal Strategy vs. Static Strategy. The MVF through point $\mathrm{C}$ is the frontier implied by a one-year surplus optimization. The frontier through point B is the frontier of a one-year surplus optimization with one rebalancing after six months (i.e. $h=2$ ). The frontier through point A is the frontier with monthly rebalancing (i.e. $h=12$ ). Finally, the frontier plotted with a straight line is the MVF implied by a strategy that rebalances daily the AL portfolio (i.e. $h=360$ ).

For the given parameter choice, the MVF are basically shifted to the left when $h$ increases. Specifically, point C, B, A, on the different frontiers show that for the given level of expected surplus the implied variance decreases from about 0.047 to about 0.042 and 0.038 , respectively, when going from a static optimization to one with 2 and 12 subperiods, respectively. Finally, the MVF implied by $h=360$ (the straight frontier in Figure 2) is very near to the one implied by $h=12$. Therefore, in this example it seems that for practical purposes a dynamic optimization involving no more than 12 subperiods would produce a mean variance performance that is essentially indistinguishable from the one obtained by an almost continuous optimal rebalancing. 


\subsection{Investment Horizon and MVF}

This section quantifies in some numerical example the dependence of the dynamically optimal AL portfolio structures on the given time horizon. We achieve this by considering an investment horizon composed by $T=36$ transaction periods and by analyzing the dynamics of the implied MFV for the terminal surplus as a function of a decreasing time horizon $T-k$ (an increasing current time $k$ ). Specifically, we focus on the changes in curvature of the MVF that are implied by the dynamically optimal strategy when the investment horizon $T-k$ shrinks to 0 .

Recall that the MVF at time $k$ for the time horizon $T$ is given by

$$
\operatorname{var}\left(S_{T}\right)=\mathcal{A}_{T-k} \cdot\left[E\left(S_{T}\right)\right]^{2}-2 \mathcal{B}_{T-k} \cdot E\left(S_{T}\right)+\mathcal{C}_{T-k}
$$

As already noted, we restrict ourselves to the discussion of the curvature parameter $\mathcal{A}_{T-k}$. Using Proposition 8 the dynamics of the curvature parameter $\mathcal{A}_{T-k}$ in terms of $\mathcal{A}_{T-s}, s \geq k$, are

$$
\mathcal{A}_{T-k}=\frac{1-\psi^{s}}{1-\psi^{k}} \mathcal{A}_{T-s}+\frac{\psi}{1-\psi} \frac{\left(1-\psi^{s}\right)\left(1-\psi^{s-1}\right)-\left(1-\psi^{k}\right)\left(1-\psi^{k-1}\right)}{\left(1-\psi^{k}\right)},
$$

where

$$
\psi=\frac{\left\langle\mathbb{1}, \mathbb{P}_{R_{T-1}^{1, \perp}}\left(R^{0}\right)\right\rangle^{2}}{\left\langle\mathbb{P}_{R_{T-1}^{1, \perp}}\left(R^{0}\right), \mathbb{P}_{R_{T-1}^{1, \perp}}\left(R^{0}\right)\right\rangle} .
$$

For the given time horizon $T$ we determine the initial curvature $\mathcal{A}_{0}$ and compute recursively $\mathcal{A}_{T-k}$ assuming $x_{T-k}=l_{T-k}=1$ and for $k=1, . ., 30$. The dynamics of the implied MVF are plotted in Figure 3 under the simplifying assumption $\mathcal{B}_{T-k}=\mathcal{B}_{0}$ and $\mathcal{C}_{T-k}=\mathcal{C}_{0}, k=1, . ., 30$.

The curvature parameter $\mathcal{A}_{T-k}$ is an increasing function of the relevant investment horizon $T-k$. Graphically, this induces in Figure 3 a flatter MVF in mean-variance space at longer horizons. Therefore, the dynamically optimal portfolio strategy perceives a less favorable mean variance trade-off at the beginning of the investment period, which becomes better as times goes on. Intuitively, this induces a more cautious portfolio structure at the beginning of the investment period, when compared with investment dates short before the final date $T$. 


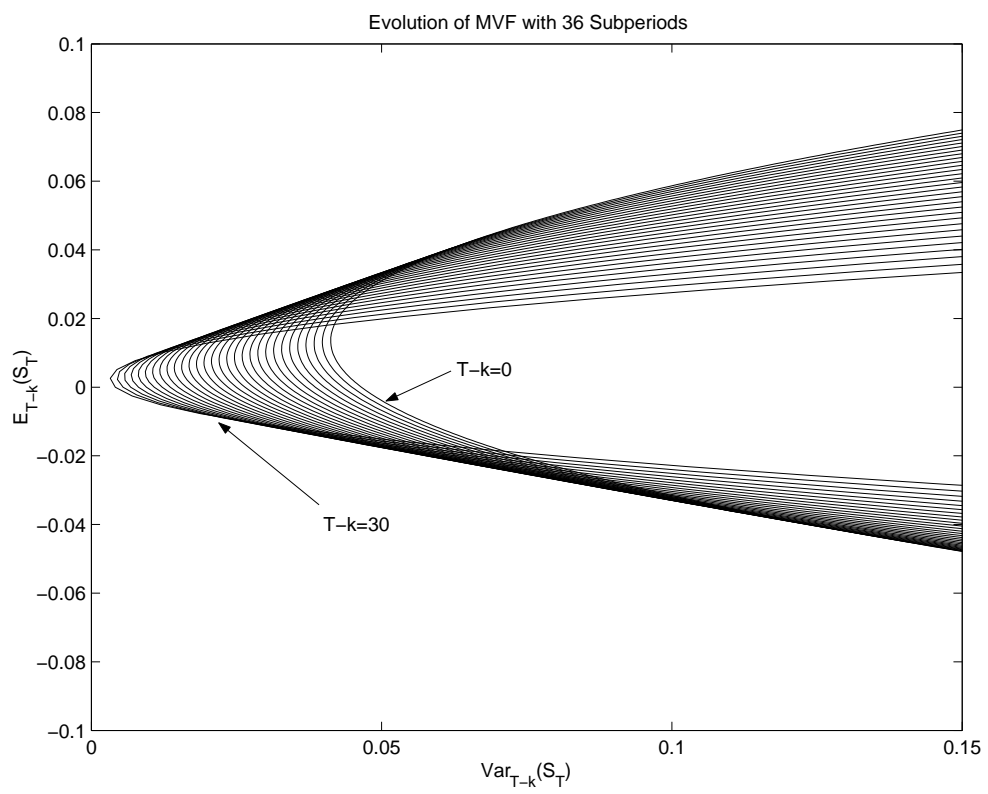

FigURE 3. Impact of the investment horizon on the curvature of the MVF. The figure shows the evolution of the MVF for a 36-period optimization problem. We only plotted the first 30 MVF.

\subsection{Iso-Moment Curves and Optimal Funding Ratios}

In Proposition 3.6 the efficient frontier depends on the initial AL structure only through the expectations:

$$
\begin{aligned}
& E\left(S_{T-k, 0}\right)=x_{T-k}\left(\left\langle\mathbb{1}, R_{T-k}^{0 e}\right\rangle-\frac{1}{f_{T-k}}\left\langle\mathbb{1}, Q_{T-k}^{0 e}\right\rangle\right), \\
& E\left(S_{T-k, 0}^{2}\right)=x_{T-k}^{2}\left(\left\langle R_{T-k}^{0 e}, R_{T-k}^{0 e}\right\rangle+\frac{1}{f_{T-k}^{2}}\left\langle Q_{T-k}^{0 e}, Q_{T-k}^{0 e}\right\rangle-\frac{2}{f_{T-k}}\left\langle R_{T-k}^{0 e}, Q_{T-k}^{0 e}\right\rangle\right),
\end{aligned}
$$

where $f_{T-k}=l_{T-k} / x_{T-k}$. Therefore, for a given level of initial assets $x_{T-k}$ the corresponding efficient frontier can be parameterized by the initial funding ratio $f_{T-k}$. On the other hand, different levels of initial assets can imply the same efficient frontier when adapting the corresponding funding ratios in an appropriate way. As a consequence, an important issue for portfolios of assets and liabilities is to characterize the optimal trade-off between initial assets and liabilities that is available to an investor with given risk aversion parameter $\gamma$. Specifically, an assets and liabilities investor can then be interested in the following "inverse" problem. Given a desired expected final surplus $E\left(S_{T}\right)$, what are the initial assets $x_{T-k}$ and 
liabilities $l_{T-k}$ necessary to obtain a minimal variance when adopting an optimal portfolio strategy? This is the issue of characterizing the optimal funding ratio for portfolios of assets and liabilities.

To answer this question it is sufficient to consider the iso-variance curves in $\left(x_{T-k}, l_{T-k}\right)$ space that are implied by a given expected final surplus or, equivalently, the implied iso-second moment curves in $\left(x_{T-k}, l_{T-k}\right)$-space. Using the orthogonal decomposition in (3.19) it follows:

$$
\begin{aligned}
E\left(S_{T}^{2}\right)= & x_{T-k}^{2}\left\langle R_{T-k}^{0 e}, R_{T-k}^{0 e}\right\rangle+l_{T-k}^{2}\left\langle Q_{T-k}^{0 e}, Q_{T-k}^{0 e}\right\rangle-2 x_{T-k} l_{T-k}\left\langle R_{T-k}^{0 e}, Q_{T-k}^{0 e}\right\rangle \\
& +\gamma^{2}\left\langle S_{T-k, e}, S_{T-k, e}\right\rangle .
\end{aligned}
$$

Consider now an investor selecting an optimal policy and note that all moments on the RHS of (4.26) are determined by the moments structure of the underlying assets and liabilities return process. The form of the iso-second moment curves implied by (4.26) depends on the second moment matrix:

$$
\Psi=\left[\begin{array}{cc}
\left\langle R_{T-k}^{0 e}, R_{T-k}^{0 e}\right\rangle & -\left\langle R_{T-k}^{0 e}, Q_{T-k}^{0 e}\right\rangle \\
-\left\langle R_{T-k}^{0 e}, Q_{T-k}^{0 e}\right\rangle & \left\langle Q_{T-k}^{0 e}, Q_{T-k}^{0 e}\right\rangle
\end{array}\right]
$$

Since $\Psi$ is positive definite, the iso-second moment curves are ellipses (for the relevant case where $R_{T-k}^{0 e}, Q_{T-k}^{0 e}$ are not perfectly correlated.). For a positive (negative) scalar product $\left\langle R_{T-k}^{0 e}, Q_{T-k}^{0 e}\right\rangle$, they are rotated counter-clockwise, (clockwise). The symmetry center of these ellipses is $(0,0)$. On the other hand, the iso-first moment curves in $\left(x_{T-k}, l_{T-k}\right)$-space are straight lines implied by level sets of the form:

$$
\left\{\left(x_{T-k}, l_{T-k}\right) \in \mathbb{R}^{2}: x_{T-k}\left\langle\mathbb{1}, R_{T-k}^{0 e}\right\rangle-l_{T-k}\left\langle\mathbb{1}, Q_{T-k}^{0 e}\right\rangle+\gamma\left\langle\mathbb{1}, S_{T-k, e}\right\rangle=\delta\right\},
$$

$\delta \in \mathbb{R}$. Hence, the optimal initial AL structure implied by a targeted expected surplus $\delta$ can be obtained geometrically as a tangency point between iso-first moment and iso-second moment curves (see Figure 4). The set of optimal initial AL structures for different surplus targets is a straight line in the $\left(x_{T-k}, l_{T-k}\right)$-space with a pendency equal to the implied optimal funding ratio, which is determined as

$$
f_{T-k}^{*}=\frac{\left\langle Q_{T-k}^{0 e}, Q_{T-k}^{0 e}\right\rangle\left\langle\mathbb{1}, R_{T-k}^{0 e}\right\rangle-\left\langle R_{T-k}^{0 e}, Q_{T-k}^{0 e}\right\rangle\left\langle\mathbb{1}, Q_{T-k}^{0 e}\right\rangle}{\left\langle R_{T-k}^{0 e}, Q_{T-k}^{0 e}\right\rangle\left\langle\mathbb{1}, R_{T-k}^{0 e}\right\rangle-\left\langle R_{T-k}^{0 e}, R_{T-k}^{0 e}\right\rangle\left\langle\mathbb{1}, Q_{T-k}^{0 e}\right\rangle} .
$$



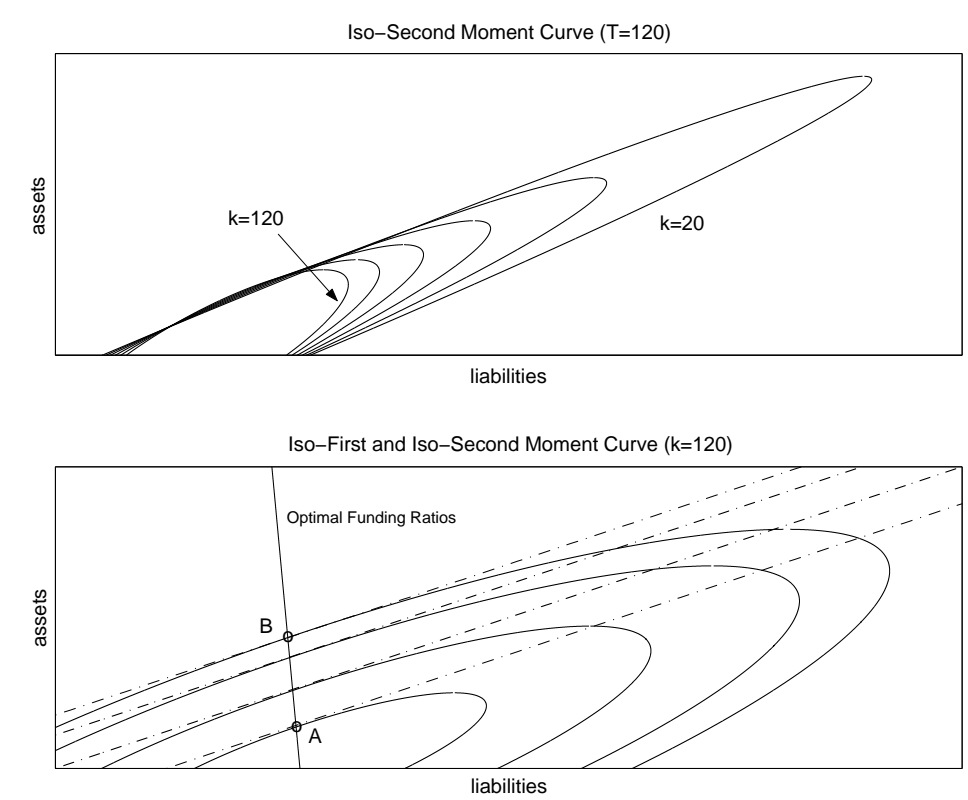

Figure 4. Iso-First Moment and Iso-Second Moment Curves. The first graph shows iso-second moment curves as a function of time $k$. The investment horizon is fixed to $T=120$. In the second graph iso-first and iso-second moment curves are plotted for a fixed $k$ but for different levels of targeted first and second moments. Point A has lower first and second moments than point B. The tangency points between iso-first and iso-second moment curves define the optimal initial AL structures and lie on a straight line.

In the i.i.d case $f_{T-k}^{*}$ can be immediately determined using Corollary 3.10.

\section{Conclusions}

We presented a geometric approach to discrete time multiperiod mean variance portfolio optimization that simplifies the mathematical analysis of these models and highlights their economic structure. We applied it to a surplus optimization problem in a multiperiod mean variance portfolio selection model including assets and liabilities. Closed form solutions have been obtained in the benchmark i.i.d. setting, which have been used to quantify specific issues like the impact of the investment horizon on the implied dynamic AL portfolio and the determination of the optimal initial funding ratio. Future research aims at incorporating in the model an endogenous liabilities optimization and some form of intertemporal constraint 
on, for instance, the downside risk of the AL surplus (cf. for example Leippold, Trojani and Trojani (2002)).

\section{Appendix}

In this appendix we provide the proofs of all propositions in the paper.

Proof of Proposition 2.1. To prove Proposition 2.1 we adopt arguments as in $\mathrm{Li}$ and Ng (2000). Convexity of $\tilde{U}$ in $E\left(S_{T}\right)$ and $E\left(S_{T}^{2}\right)$ is obvious. To prove 2., assume that $\phi^{*}$ solves (P3) and that it is not a solution of $(\mathrm{P} 4)$ for the parameter choice $\left(d\left(\phi^{*}, w\right), w\right)$. There then exists a policy $\phi$ such that

$$
-w E\left(S_{T}^{2}(\phi)\right)+d\left(\phi^{*}, w\right) E\left(S_{T}(\phi)\right)>-w E\left(S_{T}^{2}\left(\phi^{*}\right)\right)+d\left(\phi^{*}, w\right) E\left(S_{T}\left(\phi^{*}\right)\right),
$$

or, in matrix notation:

$$
\left(-w, d\left(\phi^{*}, w\right)\right)\left(\begin{array}{c}
E\left(S_{T}^{2}(\phi)\right) \\
E\left(S_{T}(\phi)\right)
\end{array}\right)>\left(-w, d\left(\phi^{*}, w\right)\right)\left(\begin{array}{c}
E\left(S_{T}^{2}\left(\phi^{*}\right)\right) \\
E\left(S_{T}\left(\phi^{*}\right)\right)
\end{array}\right)
$$

By convexity of $\tilde{U}$ it then follows

$$
\begin{aligned}
& \tilde{U}\left(E \left(S_{T}^{2}(\phi), E\left(S_{T}(\phi)\right)-\tilde{U}\left(E \left(S_{T}^{2}\left(\phi^{*}\right), E\left(S_{T}\left(\phi^{*}\right)\right)\right.\right.\right.\right. \\
\geq & \nabla \tilde{U}\left(E \left(S_{T}^{2}\left(\phi^{*}\right), E\left(S_{T}\left(\phi^{*}\right)\right)\left[\left(\begin{array}{c}
E\left(S_{T}^{2}(\phi)\right) \\
E\left(S_{T}(\phi)\right)
\end{array}\right)-\left(\begin{array}{c}
E\left(S_{T}^{2}\left(\phi^{*}\right)\right) \\
E\left(S_{T}\left(\phi^{*}\right)\right)
\end{array}\right)\right],\right.\right.
\end{aligned}
$$

denoting by $\nabla$ the gradient operator. Since for strategy $\phi^{*}$ it follows $\frac{\partial \tilde{U}}{\partial E\left(S_{T}^{2}\right)}=-w$ and $\frac{\partial \tilde{U}}{\partial E\left(S_{T}\right)}=d\left(\phi^{*}, w\right)$, the gradient $\nabla \tilde{U}$ equals $\left(-w, d\left(\phi^{*}, w\right)\right)$. Hence:

$$
\begin{aligned}
& \tilde{U}\left(E \left(S_{T}^{2}(\phi), E\left(S_{T}(\phi)\right)-\tilde{U}\left(E \left(S_{T}^{2}\left(\phi^{*}\right), E\left(S_{T}\left(\phi^{*}\right)\right)\right.\right.\right.\right. \\
\geq & \left(-w, d\left(\phi^{*}, w\right)\right)\left[\left(\begin{array}{c}
E\left(S_{T}^{2}(\phi)\right) \\
E\left(S_{T}(\phi)\right)
\end{array}\right)-\left(\begin{array}{c}
E\left(S_{T}^{2}\left(\phi^{*}\right)\right) \\
E\left(S_{T}\left(\phi^{*}\right)\right)
\end{array}\right)\right] \\
> & 0 .
\end{aligned}
$$

Therefore, $\phi^{*}$ can not be an optimal policy for (P3), a contradiction. This proves 2 . To prove 3., denote Problem (P4) by $A(\lambda, w)$, making the dependence on $(\lambda, w)$ explicit. Furthermore, define

$$
\Phi_{A}(\lambda, w)=\{\phi \mid \phi \text { is a solution of } A(\lambda, w)\} .
$$


For fixed $w$, the union $\bigcup_{\lambda} \Phi_{A}(\lambda, w)$ is the set of all solutions to (P4) parameterized by $\lambda$. In other words, $\bigcup_{\lambda} \Phi_{A}(\lambda, w)$ equals the union over $\lambda$ of all solutions $\phi(\lambda, w)$ of $(\mathrm{P} 4)$. As a consequence, by 2 of the proposition, a solution $\tilde{\phi}$ of (P3) can be always expressed as

$$
\tilde{\phi}=\tilde{\phi}(\bar{\lambda}) \in \bigcup_{\lambda} \Phi_{A}(\lambda, w), \bar{\lambda}=d(\tilde{\phi}, w) .
$$

Hence, $\tilde{\phi}$ can be also equivalently obtained by solving the problem:

$$
\begin{aligned}
& \max _{\lambda} \tilde{U}\left(E\left(S_{T}^{2}(\lambda, w)\right), E\left(S_{T}(\lambda, w)\right)\right) \\
= & \max _{\lambda}\left[-w E\left(S_{T}^{2}(\lambda, w)\right)+w\left(E\left(S_{T}(\lambda, w)\right)^{2}\right]+E\left(S_{T}(\lambda, w)\right) .\right.
\end{aligned}
$$

The first-order conditions for the optimal $\lambda^{*}$ (say) are:

$$
-w \frac{\left.\partial E\left(S_{T}^{2}\left(\lambda^{*}, w\right)\right)\right)}{\partial \lambda}+\left(1+2 w E\left(S_{T}^{*}\left(\phi^{*}\right)\right)\right) \frac{\partial E\left(S_{T}\left(\lambda^{*}, w\right)\right)}{\partial \lambda}=0,
$$

given $\phi^{*} \in \Phi_{A}(\lambda, w)$. On the other hand, if $\phi^{*} \in \Phi_{A}(\lambda, w)$, the optimality condition for (P4) gives:

$$
-w \frac{\partial E\left(S_{T}^{2}\left(\lambda^{*}, w\right)\right)}{\partial \lambda}+\lambda^{*} \frac{\partial E\left(S_{T}\left(\lambda^{*}, w\right)\right)}{\partial \lambda}=0 .
$$

These two optimality conditions imply proportionality of the vectors $\left(-w,\left(1+2 w E\left(S_{T}^{*}\left(\phi^{*}\right)\right)\right)\right.$ and $\left(-w, \lambda^{*}\right)$. This gives the condition for $\lambda^{*}$ in 3 , concluding the proof of Proposition 1.

Proof of Proposition 3.2. We first rewrite the problem in matrix form using the notations (3.7) and $e_{1}^{\prime}=\left(\begin{array}{ll}1 & 0\end{array}\right)$. This gives the problem:

$$
\left\{\begin{array}{c}
\max _{d} E\left[\gamma \mathbf{I}^{\prime} z_{T}-\frac{1}{2} z_{T}^{\prime} \mathbf{I I}^{\prime} z_{T}\right] \\
\text { s.t. } \mathbf{I}^{\prime} z_{t+1}=\mathbf{I}^{\prime} D_{t} z_{t}+\mathbf{I}^{\prime} G_{t} d_{t}, d_{t}=u_{t} e_{1}, t=0,1, \ldots, T-1
\end{array} .\right.
$$

The Bellman principle for $T-1$ implies the optimization problem (omitting for simplicity current time indices $T-1$ and writing $E(\cdot)$ for $\left.E_{T-1}(\cdot)\right)$

$$
\max _{u} E\left[\gamma \mathbf{I}^{\prime}\left(D z+G e_{1} u\right)-\frac{1}{2}\left(D z+G e_{1} u\right)^{\prime} \mathbf{I I}^{\prime}\left(D z+G e_{1} u\right)\right],
$$

taking into account the constraint $d^{\prime}=\left(\begin{array}{ll}u & 0\end{array}\right)$. A maximization with respect to $u$ gives:

$$
d^{*}=\gamma \frac{E\left(e_{1}^{\prime} G^{\prime} \mathbf{I}\right)}{E\left[e_{1}^{\prime} G^{\prime} \mathbf{I I}^{\prime} G e_{1}\right]} e_{1}-\frac{E\left(e_{1}^{\prime} G^{\prime} \mathbf{I} \mathbf{I}^{\prime} D z\right)}{E\left[e_{1}^{\prime} G^{\prime} \mathbf{I I}^{\prime} G e_{1}\right]} e_{1} .
$$


Hence, the optimal surplus at time $T$ is decomposed as a linear combination of two $L_{2^{-}}$ orthogonal pay-offs, that we denote by $S^{0 e}$ and $R^{1 e}$, respectively:

$$
S_{T}=\mathbf{I}^{\prime}\left(D z+G d^{*}\right)=\underbrace{\mathbf{I}^{\prime}\left(D z-\frac{E\left(e_{1}^{\prime} G^{\prime} \mathbf{I} \mathbf{I}^{\prime} D z\right)}{E\left[e_{1}^{\prime} G^{\prime} \mathbf{I I}^{\prime} G e_{1}\right]} G e_{1}\right)}_{S^{0 e}}+\gamma \underbrace{\frac{E\left(e_{1}^{\prime} G^{\prime} \mathbf{I}\right)}{E\left[e_{1}^{\prime} G^{\prime} \mathbf{I} \mathbf{I}^{\prime} G e_{1}\right]} \mathbf{I}^{\prime} G e_{1}}_{R^{1 e}},
$$

where

$$
S^{0 e}=\mathbf{I}^{\prime} D z-\frac{E\left(e_{1}^{\prime} G^{\prime} \mathbf{I I}^{\prime} D z\right)}{E\left[e_{1}^{\prime} G^{\prime} \mathbf{I} \mathbf{I}^{\prime} G e_{1}\right]} \mathbf{I}^{\prime} G e_{1}=R^{0} x-Q^{0} l-R^{1} \frac{E\left(R^{1} R^{0}\right) x-E\left(R^{1} Q^{0}\right) l}{E\left[\left(R^{1}\right)^{2}\right]}=\mathbf{I}^{\prime} D^{e} z,
$$

with:

$$
D^{e}=\left[\begin{array}{cc}
R^{0 e} & 0 \\
0 & Q^{0 e}
\end{array}\right], R^{0 e}=R^{0}-\frac{E\left(R^{1} R^{0}\right)}{E\left[\left(R^{1}\right)^{2}\right]} R^{1}, Q^{0 e}=Q^{0}-\frac{E\left(R^{1} Q^{0}\right)}{E\left[\left(R^{1}\right)^{2}\right]} R^{1} .
$$

Remark that the returns $R^{0 e}, Q^{0 e}, R^{1 e}$ are unaffected by the value of the state vector $z$ while the "surplus" $S^{0 e}$ is. For the value function at time $T-1$ it follows (denoting by $\langle\cdot, \cdot\rangle$ the $T-1$-conditional scalar product in $\left.L_{2}\right)$ :

$$
\begin{aligned}
J(z) & =E\left[\gamma \mathbf{I}^{\prime}\left(D z+G e_{1} u^{*}\right)-\frac{1}{2}\left(D z+G e_{1} u^{*}\right)^{\prime} \mathbf{I I}^{\prime}\left(D z+G e_{1} u^{*}\right)\right] \\
& =\gamma\left[\left\langle\mathbb{1}, S^{0 e}\right\rangle+\gamma\left\langle\mathbb{1}, R^{1 e}\right\rangle\right]-\frac{1}{2}\left[\gamma^{2}\left\langle R^{1 e}, R^{1 e}\right\rangle+\left\langle S^{0 e}, S^{0 e}\right\rangle\right] \\
& =\frac{\gamma^{2}}{2}\left\langle\mathbb{1}, R^{1 e}\right\rangle+\gamma\left\langle\mathbb{1}, S^{0 e}\right\rangle-\frac{1}{2}\left\langle S^{0 e}, S^{0 e}\right\rangle \\
& =\frac{\gamma^{2}}{2} \mathbf{I}^{\prime} C \mathbf{I}+\gamma \mathbf{I}^{\prime} B z-\frac{1}{2} z^{\prime} A z,
\end{aligned}
$$

where in the third equality we used the identity

$$
\left\langle R^{1 e}, R^{1 e}\right\rangle=\frac{E\left(e_{1}^{\prime} G^{\prime} \mathbf{I}\right) E\left(\mathbf{I}^{\prime} G e_{1}\right)}{E\left[e_{1}^{\prime} G^{\prime} \mathbf{I} \mathbf{I}^{\prime} G e_{1}\right]}=\left\langle\mathbb{1}, R^{1 e}\right\rangle,
$$

and $A, B, C$, are given by:

$$
A=E\left(D^{e \prime} \mathbf{I I}^{\prime} D^{e}\right), B=E\left(D^{e}\right), C=\frac{1}{E\left[e_{1}^{\prime} G^{\prime} \mathbf{I I}^{\prime} G e_{1}\right]} E\left(G e_{1}\right) E\left(e_{1}^{\prime} G^{\prime}\right) .
$$

For the value function at time $T-2$ it follows (omitting current time indices $T-2$ and writing $E(\cdot)$ for $\left.E_{T-2}(\cdot)\right)$ :

$J(z)=\max _{u} E\left[\mathbf{I}^{\prime} C_{T-1} \mathbf{I}+\gamma \mathbf{I}^{\prime} D_{T-1}^{e}\left(D z+u G e_{1}\right)-\frac{1}{2}\left(D z+u G e_{1}\right)^{\prime} D_{T-1}^{e \prime} \mathbf{I I}^{\prime} D_{T-1}^{e}\left(D z+u G e_{1}\right)\right]$ 
using the law of iterated expectations. Optimizing with respect to $u$ gives the optimal policy

$$
\begin{aligned}
d^{*} & =\frac{\gamma E\left(e_{1}^{\prime} G^{\prime} D_{T-1}^{e \prime} \mathbf{I}\right)}{E\left[e_{1}^{\prime} G^{\prime} D_{T-1}^{e \prime} \mathbf{I I}^{\prime} D_{T-1}^{e} G e_{1}\right]} e_{1}-\frac{E\left(e_{1}^{\prime} G^{\prime} D_{T-1}^{e \prime} \mathbf{I I}^{\prime} D_{T-1}^{e} D z\right)}{E\left[e_{1}^{\prime} G^{\prime} D_{T-1}^{e \prime} \mathbf{I} \mathbf{I}^{\prime} D_{T-1}^{e} G e_{1}\right]} e_{1} \\
& =\frac{\gamma E\left(e_{1}^{\prime}\left(D_{T-1}^{e} G\right)^{\prime} \mathbf{I}\right)}{E\left[e_{1}^{\prime}\left(D_{T-1}^{e} G\right)^{\prime} \mathbf{I I}^{\prime} D_{T-1}^{e} G e_{1}\right]} e_{1}-\frac{E\left(e_{1}^{\prime}\left(D_{T-1}^{e} G\right)^{\prime} \mathbf{I I}^{\prime} D_{T-1}^{e} D z\right)}{E\left[e_{1}^{\prime}\left(D_{T-1}^{e} G\right)^{\prime} \mathbf{I I}^{\prime} D_{T-1}^{e} G e_{1}\right]} e_{1}
\end{aligned}
$$

As a consequence, the return

$$
S_{T-1}^{0 e}=\mathbf{I}^{\prime} D_{T-1}^{e} z_{T-1}=\mathbf{I}^{\prime} D_{T-1}^{e}\left(D z+G d^{*}\right)
$$

can be decomposed as a further linear combination of two $L_{2}$-orthogonal pay-offs (that we denote again by $S^{0 e}$ and $R^{1 e}$, respectively):

$$
S_{T-1}^{0 e}=S^{0 e}+\gamma R^{1 e},
$$

with

and

$$
R^{1 e}=\frac{E\left(e_{1}^{\prime}\left(D_{T-1}^{e} G\right)^{\prime} \mathbf{I}\right)}{E\left[e_{1}^{\prime}\left(D_{T-1}^{e} G\right)^{\prime} \mathbf{I} \mathbf{I}^{\prime} D_{T-1}^{e} G e_{1}\right]} \mathbf{I}^{\prime} D_{T-1}^{e} G e_{1}
$$

$$
\begin{aligned}
S^{0 e} & =\mathbf{I}^{\prime} D_{T-1}^{e}\left(D z-G \frac{E\left(e_{1}^{\prime}\left(D_{T-1}^{e} G\right)^{\prime} \mathbf{I I}^{\prime} D_{T-1}^{e} D z\right)}{E\left[e_{1}^{\prime}\left(D_{T-1}^{e} G\right)^{\prime} \mathbf{I I}^{\prime} D_{T-1}^{e} G e_{1}\right]} e_{1}\right) \\
& =\mathbf{I}^{\prime} D_{T-1}^{e} D z-\mathbf{I}^{\prime} D_{T-1}^{e} G e_{1} \frac{E\left(e_{1}^{\prime}\left(D_{T-1}^{e} G\right)^{\prime} \mathbf{I I}^{\prime} D_{T-1}^{e} D\right)}{E\left[e_{1}^{\prime}\left(D_{T-1}^{e} G\right)^{\prime} \mathbf{I I}^{\prime} D_{T-1}^{e} G e_{1}\right]} z \\
& =R_{T-1}^{0 e} R^{0} x-Q_{T-1}^{0 e} Q^{0} x-R_{T-1}^{0 e} R^{1} \frac{E\left(R_{T-1}^{0 e} R^{1} R_{T-1}^{0 e} R^{0}\right) x-E\left(R_{T-1}^{0 e} R^{1} Q_{T-1}^{0 e} Q^{0}\right) l}{E\left[\left(R_{T-1}^{0 e} R^{1}\right)^{2}\right]} \\
& =\mathbf{I}^{\prime} D^{e} z,
\end{aligned}
$$

where:

$$
D^{e}=\left[\begin{array}{cc}
R^{0 e} & 0 \\
0 & Q^{0 e}
\end{array}\right]
$$

with

$R^{0 e}=R_{T-1}^{0 e} R^{0}-\frac{E\left(R_{T-1}^{0 e} R^{1} R_{T-1}^{0 e} R^{0}\right)}{E\left[\left(R_{T-1}^{0 e} R^{1}\right)^{2}\right]} R_{T-1}^{0 e} R^{1}, Q^{0 e}=Q_{T-1}^{0 e} Q^{0}-\frac{E\left(R_{T-1}^{0 e} R^{1} Q_{T-1}^{0 e} Q^{0}\right)}{E\left[\left(R^{1}\right)^{2}\right]} R_{T-1}^{0 e} R^{1}$.

Hence, the value function for time $T-2$ is

$$
J(z)=E\left[\mathbf{I}^{\prime} C_{T-1} \mathbf{I}+\gamma \mathbf{I}^{\prime} D^{e} z-\frac{1}{2} z^{\prime} D^{e \prime} \mathbf{I} \mathbf{I}^{\prime} D^{e} z\right]=\frac{\gamma^{2}}{2} \mathbf{I}^{\prime} C \mathbf{I}+\gamma \mathbf{I}^{\prime} B z-\frac{1}{2} z^{\prime} A z,
$$


where

$$
A=E\left(D^{e \prime} \mathbf{I I}^{\prime} D^{e}\right), B=E\left(D^{e}\right), C=C_{T-1}+\frac{1}{E\left[e_{1}^{\prime} G^{\prime} A G e_{1}\right]} B E\left(G e_{1}\right) E\left(e_{1}^{\prime} G^{\prime}\right) B^{\prime} .
$$

The expression for $C$ is derived from the same type of identity as in (6.29):

$$
\left\langle R^{1 e}, R^{1 e}\right\rangle=\frac{E\left(e_{1}^{\prime}\left(D_{T-1}^{e} G\right)^{\prime} \mathbf{I}\right) E\left(\mathbf{I}^{\prime} D_{T-1}^{e} G e_{1}\right)}{E\left[e_{1}^{\prime}\left(D_{T-1}^{e} G\right)^{\prime} \mathbf{I I}^{\prime} D_{T-1}^{e} G e_{1}\right]}=\left\langle\mathbb{1}, R^{1 e}\right\rangle,
$$

with $\langle\cdot, \cdot\rangle$ denoting the $T-2$-conditional scalar product in $L_{2}$. Therefore, $J_{T-2}$ is of the same functional form as $J_{T-1}$ and the optimal rules for $T-k, k>2$, follow recursively in the same way as for $T-2$. This concludes the proof of the proposition.

Proof Proposition 3.5. Clearly, a final surplus $S_{T}$ has minimum variance $\operatorname{var}\left(S_{T}\right)$ for a targeted expected value $E\left(S_{T}\right)$ if and only if it has minimum second moments $E\left(S_{T}^{2}\right)$ for the same targeted expected value. Using the orthogonal decomposition (3.11) it then follows for any surplus $S_{T}$ on the MVF:

$$
\begin{aligned}
& E\left(S_{T}\right)=E\left(S_{T-1,0}\right)+\gamma E\left(R_{T-1}^{1 e}\right), \\
& E\left(S_{T}^{2}\right)=E\left(S_{T-1,0}^{2}\right)+\gamma^{2} E\left[\left(R_{T-1}^{1 e}\right)^{2}\right] .
\end{aligned}
$$

Solving for $\gamma$ it follows:

$$
\left(\frac{E\left(S_{T}\right)-E\left(S_{T-1,0}\right)}{E\left(R_{T-1}^{1 e}\right)}\right)^{2}=\frac{E\left(S_{T}^{2}\right)-E\left(S_{T-1,0}^{2}\right)}{E\left[\left(R_{T-1}^{1 e}\right)^{2}\right]}=\frac{\operatorname{var}\left(S_{T}\right)+\left[E\left(S_{T}\right)\right]^{2}-E\left(S_{T-1,0}^{2}\right)}{E\left[\left(R_{T-1}^{1 e}\right)^{2}\right]} .
$$

Noting that

we finally get:

$$
\frac{E\left[\left(R_{T-1}^{1 e}\right)^{2}\right]}{\left[E\left(R_{T-1}^{1 e}\right)\right]^{2}}=\frac{1}{E\left(R_{T-1}^{1 e}\right)}
$$

$$
\begin{aligned}
\operatorname{var}\left(S_{T}\right) & =\frac{\left(E\left(S_{T}\right)-E\left(S_{T-1,0}\right)\right)^{2}}{E\left(R_{T-1}^{1 e}\right)}-\left[E\left(S_{T}\right)\right]^{2}+E\left(S_{T-1,0}^{2}\right) \\
& =\left(\frac{1}{E\left(R_{T-1}^{1 e}\right)}-1\right)\left[E\left(S_{T}\right)\right]^{2}-2 \frac{E\left(S_{T-1,0}\right)}{E\left(R_{T-1}^{1 e}\right)} E\left(S_{T}\right)+\frac{\left[E\left(S_{T-1,0}\right)\right]^{2}}{E\left(R_{T-1}^{1 e}\right)}+E\left(S_{T-1,0}^{2}\right) .
\end{aligned}
$$

This concludes the proof of the proposition. 
Proof of Proposition 3.6. The proposition is proved with conceptually identic arguments as for Proposition 3.5. Using the orthogonal decomposition (3.17) it follows for any surplus $S_{T}$ on the MVF:

$$
\begin{aligned}
& E\left(S_{T}\right)=E\left(S_{T-k, 0}\right)+\gamma E\left(S_{T-k, e}\right) \\
& E\left(S_{T}^{2}\right)=E\left(S_{T-k, 0}^{2}\right)+\gamma^{2} E\left(S_{T-k, e}^{2}\right) .
\end{aligned}
$$

Solving for $\gamma$ it follows:

$$
\left(\frac{E\left(S_{T}\right)-E\left(S_{T-k, 0}\right)}{E\left(S_{T-k, e}\right)}\right)^{2}=\frac{E\left(S_{T}^{2}\right)-E\left(S_{T-k, 0}^{2}\right)}{E\left(S_{T-k, e}^{2}\right)}=\frac{\operatorname{var}\left(S_{T}\right)+\left[E\left(S_{T}\right)\right]^{2}-E\left(S_{T-k, 0}^{2}\right)}{E\left(S_{T-k, e}^{2}\right)} .
$$

Orthogonality of $\left\{R_{T-1}^{1 e}, R_{T-2}^{1 e}, \ldots, R_{T-k}^{1 e}\right\}$ and the identities $E\left(R_{T-i}^{1 e}\right)=E\left[\left(R_{T-i}^{1 e}\right)^{2}\right], i=$ $1, . ., k$, imply:

$$
\frac{E\left(S_{T-2, e}^{2}\right)}{\left(E\left(S_{T-k, e}\right)\right)^{2}}=\frac{E\left[\left(\sum_{i=1}^{k} R_{T-i}^{1 e}\right)^{2}\right]}{\left(\sum_{i=1}^{k} E\left(R_{T-i}^{1 e}\right)\right)^{2}}=\frac{\sum_{i=1}^{k} E\left(R_{T-i}^{1 e}\right)}{\left(\sum_{i=1}^{k} E\left(R_{T-i}^{1 e}\right)\right)^{2}}=\frac{1}{E\left(S_{T-k, e}\right)} .
$$

Hence:

$$
\begin{aligned}
\operatorname{var}\left(S_{T}\right) & =\frac{\left(E\left(S_{T}\right)-E\left(S_{T-k, 0}\right)\right)^{2}}{E\left(S_{T-k, e}\right)}-\left(\left[E\left(S_{T}\right)\right]^{2}-E\left(S_{T-k, 0}^{2}\right)\right) \\
& =\left(\frac{1}{E\left(S_{T-k, e}\right)}-1\right)\left[E\left(S_{T}\right)\right]^{2}-2 \frac{E\left(S_{T-k, 0}\right)}{E\left(S_{T-k, e}\right)} E\left(S_{T}\right)+\frac{\left[E\left(S_{T-k, 0}\right)\right]^{2}}{E\left(S_{T-k, e}\right)}+E\left(S_{T-k, 0}^{2}\right)
\end{aligned}
$$

as stated. This concludes the proof of the proposition.

Proof of Proposition 3.7. We have under the i.i.d. assumption:

$$
\frac{\left\langle R_{T-k+1}^{0 e} R_{T-k}^{1}, R_{T-k+1}^{0 e} R_{T-k}^{0}\right\rangle}{\left\langle R_{T-k+1}^{0 e} R_{T-k}^{0}, R_{T-k+1}^{0 e} R_{T-k}^{0}\right\rangle}=\frac{\left\langle R_{T-k+1}^{0 e}, R_{T-k+1}^{0 e}\right\rangle}{\left\langle R_{T-k+1}^{0 e}, R_{T-k+1}^{0 e}\right\rangle} \frac{\left\langle R_{T-1}^{1}, R_{T-1}^{0}\right\rangle}{\left\langle R_{T-1}^{1}, R_{T-1}^{1}\right\rangle}=\frac{\left\langle R_{T-1}^{1}, R_{T-1}^{0}\right\rangle}{\left\langle R_{T-1}^{1}, R_{T-1}^{1}\right\rangle} .
$$

Hence, for $R_{T-k}^{0 e}$ :

$$
R_{T-k}^{0 e}=\left(R_{T-k}^{0}-\frac{\left\langle R_{T-1}^{1}, R_{T-1}^{0}\right\rangle}{\left\langle R_{T-1}^{1}, R_{T-1}^{1}\right\rangle} R_{T-k}^{1}\right) R_{T-k+1}^{0 e}=\mathbb{P}_{R_{T-k}^{1, \perp}}\left(R^{0}\right) R_{T-k+1}^{0 e}=\prod_{i=0}^{k-1} \mathbb{P}_{R_{T-k+i}^{1, \perp}}\left(R^{0}\right)
$$


Similarly for $R_{T-k+i}^{1 e}$, using this last result:

$$
\begin{aligned}
R_{T-k+i}^{1 e} & =\frac{\left\langle\mathbb{1}, R_{T-k+i}^{1} R_{T-k+i+1}^{0 e}\right\rangle}{\left\langle R_{T-k+i}^{1} R_{T-k+i+1}^{0 e}, R_{T-k+i}^{1} R_{T-k+i+1}^{0 e}\right\rangle} R_{T-k+i}^{1} R_{T-k+i+1}^{0 e} \\
& =\frac{\left\langle\mathbb{1}, R_{T-1}^{1}\right\rangle}{\left\langle R_{T-1}^{1}, R_{T-1}^{1}\right\rangle} R_{T-k+i}^{1} \frac{\left\langle\mathbb{1}, R_{T-k+i+1}^{0 e}\right\rangle}{\left\langle R_{T-k+i+1}^{0 e}, R_{T-k+i+1}^{0 e}\right\rangle} R_{T-k+i+1}^{0 e} \\
& =\mathbb{P}_{R_{T-k+i}^{1}}(\mathbb{l}) \prod_{j=i+1}^{k-1} \mathbb{P}_{\mathbb{P}_{R_{T-1}^{1, \perp}}\left(R^{0}\right)}(\mathbb{l}) .
\end{aligned}
$$

For $Q_{T-k}^{0 e}$ we first have:

$$
\begin{aligned}
Q_{T-k}^{0 e} & =\mathbb{P}_{\left(R_{T-k}^{1} R_{T-k+1}^{0 e}\right)^{\perp}}\left(Q_{T-k}^{0} Q_{T-k+1}^{0 e}\right) \\
& =Q_{T-k}^{0} Q_{T-k+1}^{0 e}-\mathbb{P}_{R_{T-k}^{1} R_{T-k+1}^{0 e}}\left(Q_{T-k}^{0} Q_{T-k+1}^{0 e}\right) \\
& =Q_{T-k}^{0} Q_{T-k+1}^{0 e}-\mathbb{P}_{R_{T-k}^{1} R_{T-k+1}^{0 e}}\left(Q_{T-k}^{0} \mathbb{P}_{\left(R_{T-k+1}^{1} R_{T-k+2}^{0 e}\right)^{\perp}}\left(Q_{T-k+1}^{0} Q_{T-k+2}^{0 e}\right)\right) \\
& =Q_{T-k}^{0} Q_{T-k+1}^{0 e}-\mathbb{P}_{R_{T-k}^{1} R_{T-k+1}^{0 e}}\left(Q_{T-k}^{0} Q_{T-k+1}^{0} Q_{T-k+2}^{0 e}\right) \\
& =Q_{T-k}^{0} Q_{T-k+1}^{0 e}-\mathbb{P}_{R_{T-k}^{1} R_{T-k+1}^{0 e}}\left(\prod_{i=0}^{k-1} Q_{T-k+i}^{0}\right),
\end{aligned}
$$

because by construction

$$
R_{T-k+1}^{0 e} \perp R_{T-k+1}^{1} R_{T-k+2}^{0 e}
$$

Further:

$$
\begin{aligned}
\mathbb{P}_{R_{T-k}^{1} R_{T-k+1}^{0 e}}\left(\prod_{i=0}^{k-1} Q_{T-k+i}^{0}\right)= & \frac{\left\langle R_{T-k}^{1} R_{T-k+1}^{0 e}, \prod_{i=0}^{k-1} Q_{T-k+i}^{0}\right\rangle}{\left\langle R_{T-k}^{1} R_{T-k+1}^{0 e}, R_{T-k}^{1} R_{T-k+1}^{0 e}\right\rangle} R_{T-k}^{1} R_{T-k+1}^{0 e} \\
= & \frac{\left\langle R_{T-k}^{1} \prod_{i=1}^{k-1} \mathbb{P}_{R_{T-k+i}^{1, \perp}}\left(R^{0}\right), \prod_{i=0}^{k-1} Q_{T-k+i}^{0}\right\rangle}{\left\langle R_{T-k}^{1} \prod_{i=1}^{k-1} \mathbb{P}_{R_{T-k+i}^{1, \perp}}\left(R^{0}\right), R_{T-k}^{1} \prod_{i=1}^{k-1} \mathbb{P}_{R_{T-k+i}^{1, \perp}}\left(R^{0}\right)\right\rangle} \\
& \times R_{T-k}^{1} \prod_{i=1}^{k-1} \mathbb{P}_{R_{T-k+i}^{1, \perp}}\left(R^{0}\right) \\
= & \mathbb{P}_{R_{T-k}^{1}}\left(Q^{0}\right) \prod_{i=1}^{k-1} \mathbb{P}_{\mathbb{P}_{R_{T-k+i}^{1, \perp}}{ }_{\left(R^{0}\right)}\left(Q^{0}\right) .}
\end{aligned}
$$

Therefore:

$$
Q_{T-k}^{0 e}=Q_{T-k}^{0} Q_{T-k+1}^{0 e}-\mathbb{P}_{R_{T-k}^{1}}\left(Q^{0}\right) \prod_{i=1}^{k-1} \mathbb{P}_{\mathbb{P}_{R_{T-k+i}^{1, \perp}}\left(R^{0}\right)}\left(Q^{0}\right)=Q_{T-k}^{0} Q_{T-k+1}^{0 e}-\mathbb{P}_{R_{T-k}^{1}}\left(Q^{0}\right) A_{T-k+1},
$$


say. Hence, $Q_{T-k}^{0 e}$ satisfies a linear first order difference equation with solution given by:

$$
\begin{aligned}
Q_{T-k}^{0 e} & =Q_{T-k}^{0} Q_{T-k+1}^{0 e}-\mathbb{P}_{R_{T-k}^{1}}\left(Q^{0}\right) A_{T-k+1} \\
& =Q_{T-k}^{0}\left(Q_{T-k+1}^{0} Q_{T-k+2}^{0 e}-\mathbb{P}_{R_{T-k+1}^{1}}\left(Q^{0}\right) A_{T-k+2}\right)-\mathbb{P}_{R_{T-k}^{1}}\left(Q^{0}\right) A_{T-k+1} \\
& =Q_{T-k}^{0} Q_{T-k+1}^{0} Q_{T-k+2}^{0 e}-Q_{T-k}^{0} \mathbb{P}_{R_{T-k+1}^{1}}\left(Q^{0}\right) A_{T-k+2}-\mathbb{P}_{R_{T-k}^{1}}\left(Q^{0}\right) A_{T-k+1} \\
& =\prod_{i=0}^{k-1} Q_{T-k+i}^{0}-\sum_{i=0}^{k-1}\left(\prod_{j=0}^{i-1} Q_{T-k+j}^{0}\right) \mathbb{P}_{R_{T-k+i}^{1}\left(R^{0}\right)}\left(Q^{0}\right) A_{T-k+1+i},
\end{aligned}
$$

giving the claim of the proposition.

Proof of Corollary 3.8-3.10. Given the i.i.d. assumption it follows for $R_{T-k}^{0 e}$ :

$$
E\left(R_{T-k}^{0 e}\right)=\prod_{i=0}^{k-1}\left\langle\mathbb{1}, \mathbb{P}_{R_{T-k+i}^{1, \perp}}\left(R^{0}\right)\right\rangle=\left\langle\mathbb{1}, \mathbb{P}_{R_{T-1}^{1, \perp}}\left(R^{0}\right)\right\rangle^{k}
$$

Further:

$$
\left\langle R_{T-k}^{0 e}, R_{T-k}^{0 e}\right\rangle=\prod_{i=0}^{k-1}\left\langle\mathbb{P}_{R_{T-k+i}^{1, \perp}}\left(R^{0}\right), \mathbb{P}_{R_{T-k+i}^{1, \perp}}\left(R^{0}\right)\right\rangle=\left\langle\mathbb{P}_{R_{T-1}^{1, \perp}}\left(R^{0}\right), \mathbb{P}_{R_{T-1}^{1, \perp}}\left(R^{0}\right)\right\rangle^{k},
$$

and

$$
E\left(R_{T-k+i}^{1 e}\right)=\left\langle\mathbb{1}, \mathbb{P}_{R_{T-k+i}^{1}}(\mathbb{l})\right\rangle \prod_{j=i+1}^{k-1}\left\langle\mathbb{1}, \mathbb{P}_{\mathbb{P}_{R_{T-k+i+j}^{1, \perp}}\left(R^{0}\right)}(\mathbb{l})\right\rangle=\left\langle\mathbb{1}, \mathbb{P}_{R_{T-1}^{1}}(\mathbb{l})\right\rangle\left\langle\mathbb{1}, \mathbb{P}_{\mathbb{P}_{R_{T-1}^{1, \perp}}\left(R^{0}\right)}(\mathbb{l})\right\rangle^{k-1}
$$

Moreover (eg. 6.30):

$$
\begin{aligned}
E\left(Q_{T-k}^{0 e}\right) & =\left\langle\mathbb{1}, Q_{T-k}^{0} Q_{T-k+1}^{0 e}-\mathbb{P}_{R_{T-k}^{1}}\left(Q^{0}\right) A_{T-k+1}\right\rangle \\
& =\left\langle\mathbb{1}, Q_{T-k}^{0}\right\rangle\left\langle\mathbb{1}, Q_{T-k+1}^{0 e}\right\rangle-\left\langle\mathbb{1}, \mathbb{P}_{R_{T-k}^{1}}\left(Q^{0}\right)\right\rangle\left\langle\mathbb{1}, A_{T-k+1}\right\rangle \\
& =\left\langle\mathbb{1}, Q_{T-k}^{0}\right\rangle^{k}-\sum_{i=0}^{k-1}\left(\prod_{j=0}^{i-1}\left\langle\mathbb{1}, Q_{T-k+j}^{0}\right\rangle\right)\left\langle\mathbb{1}, \mathbb{P}_{R_{T-k+i}^{1}}\left(Q^{0}\right)\right\rangle\left\langle\mathbb{1}, A_{T-k+i+1}\right\rangle \\
& =\left\langle\mathbb{1}, Q_{T-1}^{0}\right\rangle^{k}-\sum_{i=0}^{k-1}\left\langle\mathbb{1}, Q_{T-1}^{0}\right\rangle^{i}\left\langle\mathbb{1}, \mathbb{P}_{R_{T-1}^{1}}\left(Q^{0}\right)\right\rangle\left\langle\mathbb{1}, A_{T-k+i+1}\right\rangle .
\end{aligned}
$$

Reminding that:

$$
\left\langle\mathbb{l}, A_{T-k+i+1}\right\rangle=\prod_{j=i+1}^{k-1}\left\langle\mathbb{1}, \mathbb{P}_{\mathbb{P}_{R_{T-k+i}^{1, \perp}}\left(R^{0}\right)}\left(Q^{0}\right)\right\rangle=\left\langle\mathbb{1}, \mathbb{P}_{\mathbb{P}_{R_{T-1}^{1, \perp}}\left(R^{0}\right)}\left(Q^{0}\right)\right\rangle^{k-i-1}
$$


the result for $E\left(Q_{T-k}^{0 e}\right)$ is obtained. Now, for $\left\langle R_{T-k}^{0 e}, Q_{T-k}^{0 e}\right\rangle$ :

$$
\begin{aligned}
\left\langle R_{T-k}^{0 e}, Q_{T-k}^{0 e}\right\rangle & =\left\langle\prod_{i=0}^{k-1} \mathbb{P}_{R_{T-k+i}^{1, \perp}}\left(R^{0}\right), Q_{T-k}^{0} Q_{T-k+1}^{0 e}-\mathbb{P}_{R_{T-k}^{1}}\left(Q^{0}\right) A_{T-k+1}\right\rangle \\
& =\left\langle\mathbb{P}_{R_{T-1}^{1, \perp}}\left(R^{0}\right), Q_{T-1}^{0}\right\rangle\left\langle R_{T-k+1}^{0 e}, Q_{T-k+1}^{0 e}\right\rangle \\
& =\left\langle\mathbb{P}_{R_{T-1}^{1, \perp}}\left(R^{0}\right), Q_{T-1}^{0}\right\rangle^{k},
\end{aligned}
$$

because

$\left\langle\prod_{i=0}^{k-1} \mathbb{P}_{R_{T-k+i}^{1, \perp}}\left(R^{0}\right), \mathbb{P}_{R_{T-k}^{1}}\left(Q^{0}\right) A_{T-k+1}\right\rangle=\left\langle\mathbb{P}_{R_{T-k}^{1, \perp}}\left(R^{0}\right), \mathbb{P}_{R_{T-k}^{1}}\left(Q^{0}\right)\right\rangle\left\langle R_{T-k+1}^{0 e}, A_{T-k+1}\right\rangle=0$.

Finally, to compute $\left\langle Q_{T-k}^{0 e}, Q_{T-k}^{0 e}\right\rangle$ we first remark (eg. again (6.30)):

$$
\begin{aligned}
\left\langle Q_{T-k+1}^{0 e}, A_{T-k+1}\right\rangle & =\left\langle Q_{T-k+1}^{0} Q_{T-k+2}^{0 e}-\mathbb{P}_{R_{T-k+1}^{1}}\left(Q^{0}\right) A_{T-k+2}, A_{T-k+1}\right\rangle \\
& =\left\langle Q_{T-k+1}^{0} Q_{T-k+2}^{0 e}, A_{T-k+1}\right\rangle,
\end{aligned}
$$

because:

$$
\left\langle\mathbb{P}_{R_{T-k+1}^{1}}\left(Q^{0}\right) A_{T-k+2}, A_{T-k+1}\right\rangle=\left\langle\mathbb{P}_{R_{T-1}^{1}}\left(Q^{0}\right), \mathbb{P}_{\mathbb{P}_{R_{T-1}^{1, \perp}}}\left(Q^{0}\right)\right\rangle\left\langle A_{T-k+2}, A_{T-k+2}\right\rangle=0 .
$$

This gives

$$
\begin{aligned}
\left\langle Q_{T-k+1}^{0 e}, A_{T-k+1}\right\rangle & =\left\langle Q_{T-k+1}^{0} Q_{T-k+2}^{0 e}, A_{T-k+1}\right\rangle \\
& =\left\langle\prod_{i=1}^{k-1} Q_{T-1}^{0}, A_{T-k+1}\right\rangle=\left\langle Q_{T-1}^{0}, \mathbb{P}_{\mathbb{P}_{R_{T-1}^{1, \perp}}}\left(Q^{0}\right)\right\rangle^{k-1}
\end{aligned}
$$

Moreover

$$
\left\langle A_{T-k+1}, A_{T-k+1}\right\rangle=\left\langle\mathbb{P}_{\mathbb{P}_{R_{T-1}^{1, \perp}}}\left(Q^{0}\right), \mathbb{P}_{\mathbb{P}_{R_{T-1}^{1, \perp}}}\left(Q^{0}\right)\right\rangle^{k-1} .
$$

Together with the identity $\left\langle\mathbb{P}_{R_{T-k}^{1}}\left(Q^{0}\right), \mathbb{P}_{R_{T-k}^{1}}\left(Q^{0}\right)\right\rangle=\left\langle Q_{T-k}^{0}, \mathbb{P}_{R_{T-k}^{1}}\left(Q^{0}\right)\right\rangle$ this yields:

$$
\begin{aligned}
\left\langle Q_{T-k}^{0 e}, Q_{T-k}^{0 e}\right\rangle= & \left\langle Q_{T-k}^{0} Q_{T-k+1}^{0 e}-\mathbb{P}_{R_{T-k}^{1}}\left(Q^{0}\right) A_{T-k+1}, Q_{T-k}^{0} Q_{T-k+1}^{0 e}-\mathbb{P}_{R_{T-k}^{1}}\left(Q^{0}\right) A_{T-k+1}\right\rangle \\
= & \left\langle Q_{T-k}^{0}, Q_{T-k}^{0}\right\rangle\left\langle Q_{T-k+1}^{0 e}, Q_{T-k+1}^{0 e}\right\rangle \\
& -\left\langle\mathbb{P}_{R_{T-k}^{1}}\left(Q^{0}\right), \mathbb{P}_{R_{T-k}^{1}}\left(Q^{0}\right)\right\rangle\left\langle A_{T-k+1}, A_{T-k+1}\right\rangle .
\end{aligned}
$$


Using the notation

$$
C=\left\langle\mathbb{P}_{\mathbb{P}_{T-1}^{1, \perp}}\left(R^{0}\right)\left(Q^{0}\right), \mathbb{P}_{\mathbb{P}_{R_{T-1}^{1, \perp}}\left(R^{0}\right)}\left(Q^{0}\right)\right\rangle,
$$

the solution to this first order difference equation is

$$
\left\langle Q_{T-k}^{0 e}, Q_{T-k}^{0 e}\right\rangle=\left\langle Q_{T-1}^{0}, Q_{T-1}^{0}\right\rangle^{k}-\left\langle\mathbb{P}_{R_{T-1}^{1}}\left(Q^{0}\right), \mathbb{P}_{R_{T-1}^{1}}\left(Q^{0}\right)\right\rangle \sum_{i=0}^{k-1}\left\langle Q_{T-1}^{0}, Q_{T-1}^{0}\right\rangle^{i} C^{k-i-1}
$$

Since:

$$
\left\langle\mathbb{P}_{R_{T-1}^{1}}\left(Q^{0}\right), \mathbb{P}_{R_{T-1}^{1}}\left(Q^{0}\right)\right\rangle=\left\langle Q^{0}, \mathbb{P}_{R_{T-1}^{1}}\left(Q^{0}\right)\right\rangle
$$

and

$$
\left\langle\mathbb{P}_{\mathbb{P}_{R_{T-1}^{1, \perp}}\left(R^{0}\right)}\left(Q^{0}\right), \mathbb{P}_{\mathbb{P}_{R_{T-1}^{1, \perp}}\left(R^{0}\right)}\left(Q^{0}\right)\right\rangle=\left\langle Q^{0}, \mathbb{P}_{\mathbb{P}_{R_{T-1}^{1, \perp}}\left(R^{0}\right)}\left(Q^{0}\right)\right\rangle,
$$

the result follows. 


\section{REFERENCES}

Chen, A., C. Jen, and S. Zionts (1971): The Optimal Portfolio Revision Policy. Journal of Business 44, 51-61.

Cvitanic, J. and I. Karatzas (1992): Convex Duality in Constrained Portfolio Optimization. Annals of Applied Probability 2, 767-818.

Grossman, S. J. and J. L. Vila (1992): Optimal Dynamic Trading with Leverage Constraints. Journal of Financial and Quantitative Analysis 27, 151-168.

Hansen, L. P. and S. F. Richard (1987): The Role of Conditioning Information in Deducing Testable Restrictions Implied by Dynamic Asset Pricing Models. Econometrica 587-613.

He, H. and H. F. PAGes (1993): Labor Income, Borrowing Constraints, and Equilibrium Asset Prices; a Duality Approach. Economic Theory 3, 663-696.

He, H. and N. D. Pearson (1991a): Consumption and Portfolio Policies with Incomplete Markets and Short Sales constraints: The Finite Dimensional Case. Mathematical Finance 1, 1-10.

He, H. and N. D. Pearson (1991b): Consumption and Portfolio Policies with Incomplete Markets and Short Sales constraints: The Infinite Dimensional Case. Journal of Economic Theory 54, 259-304.

Karatzas, I., J. Lehoczky, S. E. Shreve, and G. L. Xu (1991): Martingale and Duality Methods for Utility Maximization in an Incomplete Market. SIAM Journal of Control and Optimization 29, 702-730.

Keel, A. and H. Müller (1995): Efficient Portfolios in the Asset Liability Context. Astin Bulletin 25, 33-48.

Leippold, M., F. Trojani, and P. Vanini (2002): Asset-Liability Management with Endogenous Liabilities. Working paper, University of Southern Switzerland.

Li, D. and W.-L. NG (2000): Optimal Dynamic Portfolio Selection: Multi-Period Mean-Variance Formulation. Mathematical Finance 10(3), 387-406.

Merton, R. (1969): Lifetime Portfolio Selection under Uncertainty: The Continuous-Time Case. Review of Economics and Statistics 51, 247-256.

Merton, R. (1971): Optimum Consumption and Portfolio Rules in a Continuous-Time Model. Journal of Economic Theory 3, 373-413.

Mossin, J. (1968): Optimal Multiperiod Portfolio Policies. Journal of Business 41, 215-229.

Samuleson, P. (1969): Lifetime Portfolio Selection by Dynamic Stochastic Programming. The Review of Economics and Statistics 50, 239-246.

Sharpe, W. F. and L. G. Tint (1990): Liabilities-A New Approach. Journal of Portfolio Management 16(2), $5-10$.

Shreve, S. and G.-L. Xu (1992a): A Duality Method for Optimal Consumption and Investment under Shortselling Prohibition, Part I: General market coefficients. Annals of Applied Probability 2, 87-112.

Shreve, S. and G.-L. Xu (1992b): A Duality Method for Optimal Consumption and Investment under Shortselling Prohibition, Part II: Constant Market Coefficients. Annals of Applied Probability 2, 314-328.

Sмith, K. (1967): A Transition Model for Portfolio Revision. Journal of Finance 22, 425-439.

Tepla, L. (1998): Optimal Portfolio Policies with Borrowing and Shortsale Constraints. Working paper, INSEAD.

Vila, J. L. and T. ZARiphopoulou (1994): Optimal Consumption and Portfolio Choice with Borrowing Constraints. Technical report, MIT Sloan.

Zariphopoulou, T. (1989): Consumption-Investment Models with Constraints. SIAM Journal of Control and Optimization 32, 59-85. 